\title{
Diversification and expression of the PIN, AUX/LAX, and ABCB families of putative auxin transporters in Populus
} \author{
Rachel Spicer ${ }^{5 *}$ \\ ${ }^{1}$ Department of Horticulture and Landscape Architecture, Purdue University, West Lafayette, IN, USA \\ 2 Rowland Institute at Harvard, Cambridge, MA, USA \\ ${ }^{3}$ Division of Invertebrate Zoology, American Museum of Natural History, New York, NY, USA \\ ${ }^{4}$ Department of Mathematics and Computer Science, Philipps University, Marburg, Germany \\ ${ }^{5}$ Department of Botany, Connecticut College, New London, CT, USA
}

Nicola Carraro ${ }^{1}$, Tracy Eizabeth Tisdale-Orr ${ }^{2}$, Ronald Matthew Clouse ${ }^{3}$, Anne Sophie Knöller ${ }^{4}$ and

Edited by:

Angus S. Murphy, Purdue University, USA

\section{Reviewed by:}

Serge Delrot, University of Bordeaux, France

Ranjan Swarup, University of Nottingham, UK

\section{*Correspondence:}

Rachel Spicer, Department of Botany, Connecticut College, 270 Mohegan Avenue, New London, CT 06320, USA.

e-mail: rspicer@conncoll.edu
Intercellular transport of the plant hormone auxin is mediated by three families of membrane-bound protein carriers, with the PIN and $A B C B$ families coding primarily for efflux proteins and the $A U X / L A X$ family coding for influx proteins. In the last decade our understanding of gene and protein function for these transporters in Arabidopsis has expanded rapidly but very little is known about their role in woody plant development. Here we present a comprehensive account of all three families in the model woody species Populus, including chromosome distribution, protein structure, quantitative gene expression, and evolutionary relationships. The PIN and AUX/LAX gene families in Populus comprise 16 and 8 members respectively and show evidence for the retention of paralogs following a relatively recent whole genome duplication. There is also differential expression across tissues within many gene pairs. The $A B C B$ family is previously undescribed in Populus and includes 20 members, showing a much deeper evolutionary history, including both tandem and whole genome duplication as well as probable gene loss. A striking number of these transporters are expressed in developing Populus stems and we suggest that evolutionary and structural relationships with known auxin transporters in Arabidopsis can point toward candidate genes for further study in Populus. This is especially important for the ABCBs, which is a large family and includes members in Arabidopsis that are able to transport other substrates in addition to auxin. Protein modeling, sequence alignment and expression data all point to ABCB1.1 as a likely auxin transport protein in Populus. Given that basipetal auxin flow through the cambial zone shapes the development of woody stems, it is important that we identify the full complement of genes involved in this process. This work should lay the foundation for studies targeting specific proteins for functional characterization and in situ localization.

Keywords: auxin, PIN, AUX/LAX, ABCB, Populus

\section{INTRODUCTION}

Plant development is highly plastic owing to growth via meristems, and this plasticity is fundamental to the ability of plants, as sessile organisms, to adapt to changing environments. Developmental flexibility is particularly important for trees, which can live for thousands of years in the same place, growing massive bodies that must face a multitude of environmental challenges. The plant hormone auxin is well established as a key regulator of plant morphogenesis and in recent years the molecular mechanisms of transport and action have been elucidated. With the publication of the Populus trichocarpa genome (Tuskan et al., 2006), new tools to improve our understanding of secondary growth - the type of vascular growth that defines woody plants - became available. Populus is not only the dominant model species for woody plant growth, but also a valuable crop for pulp, bioenergy production, and carbon sequestration. Thus, understanding the mechanisms that underlie auxin transport in Populus is of interest both in the context of the evolution of plant development and as a means to manipulate plant architecture, biomass production, and fiber quality.

The auxins as a group include several molecules, with the most abundant natural form in plants being indole-3-acetic acid (IAA). Auxin synthesis occurs in young, actively growing tissues including shoot tips, young leaves, and germinating seeds (Ljung et al., 2001a,b), and increasing evidence suggests that synthesis takes place in the roots as well (Ljung et al., 2005). Auxin moves from the sites of production throughout the plant via two routes: long distance transport of conjugated forms in the phloem and short distance transport of "free" (non-conjugated) auxin via polar auxin transport (PAT). By far the better studied route, PAT is a form of active intercellular transport mediated by proteins inserted in the plasma membrane that belong to three distinct families. The PIN and $A B C B$ families encode efflux proteins (i.e., proteins that facilitate movement out of cells), whereas members of the 
$A U X / L A X$ family facilitate auxin entry into cells, along with passive diffusion. PAT is relatively slow $(5-20 \mathrm{~mm} / \mathrm{h}$; Lomax et al., 1995), saturable and can be impaired by the application of both competitive inhibitors and inhibitors of protein synthesis (Katekar and Geissler, 1980; Sussman and Goldsmith, 1981). This form of transport is considered polar because the protein carriers are often asymmetrically positioned in the plasma membrane such that transport is directional. Transport directionality can then be altered on relatively short timescales in response to repositioning of the protein carriers. Feedback mechanisms also exist such that PAT is often self-reinforcing, with multiple transport proteins themselves being upregulated by auxin (Sauer et al., 2006; Titapiwatanakun and Murphy, 2009).

The PIN proteins have been studied extensively in Arabidopsis thaliana (Chen et al., 1998; Luschnig et al., 1998; Müller et al., 1998; Utsuno et al., 1998; Friml et al., 2002a,b, 2003) and show dynamic polar localization at the plasma membrane (PIN1, PIN2, PIN3, PIN7) or in the endoplasmic reticulum (ER) (PIN5, PIN6, PIN8; Mravec et al., 2009; Friml and Jones, 2010). PIN1 was first described as mediating PAT and determining organ outgrowth at the inflorescence (Okada et al., 1991; Gälweiler et al., 1998; Vernoux et al., 2011). Subsequently its role in embryogenesis, vein patterning, vascular development, and root development were established (Friml et al., 2003; Vieten et al., 2005; Scarpella et al., 2006; Petrásek and Friml, 2009). The characterization of PIN genes has been expanded to include the monocotyledons Zea mays and Oryza sativa, both of which express several PINs thought to be specific to the monocots. In maize, $\mathrm{ZmPIN} 1 \mathrm{a}, \mathrm{b}$, and $\mathrm{c}$ are responsible for directing auxin transport in the male and female inflorescences and in the floret meristems (Carraro et al., 2006; Wu and McSteen, 2007). They are also involved in endosperm and embryonic development (Forestan et al., 2010) and in the maintenance of phyllotaxy (Lee et al., 2009). The monocot-specific PINs from rice (OsPIN9, OsPIN10a, and OsPIN10b) are highly expressed in adventitious root primordia and pericycle cells at the stem-base, suggesting that they may have evolved to promote adventitious root development (Wang et al., 2009).

Members of the AUXIN/LIKE AUXIN (AUX/LAX) family in Arabidopsis (Bennett et al., 1996; Yemm et al., 2004) are largely responsible for auxin influx, although the protonated form of auxin (IAAH) is able to passively diffuse into cells. The founder member $A U X 1$ encodes a plasma membrane protein that belongs to the amino acid permease family of proton-driven transporters and functions as an anionic symporter (Swarup et al., 2005; Yang et al., 2006). AUX1-mediated IAA uptake is implicated in gravitropic response, as the agravitropic phenotype of the auxl mutant can be phenocopied in wild-type seedlings by applying the auxin influx carrier inhibitor 1-naphthoxyaceticacids (1-NOA) and rescued using the membrane-permeable auxin 1-naphthaleneacetic acid (NAA; Swarup et al., 2001; Yemm et al., 2004). The paralogs of $A U X 1, L A X 1, L A X 2$, and $L A X 3$ encode proteins that maintain a correct phyllotactic pattern at the shoot apical meristem (SAM), as they act together with PIN1-mediated auxin efflux (Bainbridge et al., 2008). LAX3 is also involved in the development of lateral root primordia (Swarup et al., 2008).

The involvement of ABCB [ATP-binding cassette (ABC) transporters of the $\mathrm{B}$ class, previously known as multidrug resistance
(MDR)/Phosphoglycoprotein (PGP)] proteins in auxin transport was first hypothesized when expression of ABCB1/PGP1 in Arabidopsis was found to regulate hypocotyl elongation in a lightdependent fashion (Sidler et al., 1998). Subsequently, ABCB1 was shown to function with ABCB19/PGP19/MDR1 in mediating PAT (Noh et al., 2001). ABCB1 and ABCB19 are the closest Arabidopsis orthologs of mammalian ABCB1-type MDR transporters and although specificity for auxin is not assured (Lee et al., 2008), some appear to transport auxin with relatively high substrate specificity (Titapiwatanakun and Murphy, 2009; Yang and Murphy, 2009). ABCB14 and ABCB15 promote auxin transport along the inflorescence of Arabidopsis, where they are expressed in vascular tissue and interfascicular fibers. Inflorescence stems in both knockout mutants show a reduction in PAT (Kaneda et al., 2011). ABCB4 from Arabidopsis is involved in basipetal PAT in the root (Terasaka et al., 2005; Wu et al., 2007; Kubeš et al., 2011) and, although most $\mathrm{ABCBs}$ studied to date function as efflux carriers, heterologous expression of $\mathrm{ABCB} 4$ suggests that it functions as an auxin influx carrier under low concentrations of IAA and reverses to efflux when IAA concentrations increase (Yang and Murphy, 2009). The $A B C B 1 / P G P 1$ ortholog has been cloned in maize (Brachytic2/ZmPGP1) and in Sorghum bicolor (dwarf3/SbPGP1) and shown to be responsible for IAA transport along the stem (Multani et al., 2003; Knöller et al., 2010).

Our understanding of PAT and its role in development has advanced considerably in Arabidopsis and to a lesser extent in monocots, but the functional significance of these transport proteins - particularly the $\mathrm{ABCBs}$ - remain largely unknown in woody plants. Woody plants are defined by the production of secondary vascular tissue, specifically secondary xylem and phloem. These vascular tissues are derived from a lateral meristem called the vascular cambium that encircles the stem, adding new cells that will ultimately differentiate into xylem toward the inside of the stem and phloem toward the outside. Given the demonstrated role of PAT in vascular development in herbaceous plants it seems logical to expect a role in secondary growth. Indeed, the vascular cambium contains high levels of IAA in both Pinus and Populus, with a peak concentration occurring either in the cambial initials themselves, or perhaps more likely, in the earliest differentiating xylem elements (Uggla et al., 1996, 1998; Tuominen et al., 1997; Hellgren et al., 2004). Concentrations rapidly decline through the regions of cell differentiation to near zero in mature secondary xylem and phloem. Auxin transport in the cambium is basipetal (Lachaud and Bonnemain, 1984; Uggla et al., 1998; Kramer et al., 2008) and several members of the PIN and AUX/LAX gene families are expressed in developing Populus stems (Schrader et al., 2003, 2004; Nilsson et al., 2008). Furthermore, expression of one or more PIN and AUX/LAX genes is downregulated with the onset of dormancy (Schrader et al., 2003, 2004) and upregulated following exogenous application of IAA and/or gibberellins (Schrader et al., 2003; Björklund et al., 2007). Despite several excellent studies in Populus, our knowledge of the molecular mechanisms that regulate PAT in woody plants is essentially restricted to the expression patterns of just three PIN and AUX/LAX genes. A more comprehensive understanding of PAT gene and protein function in Populus will help to clarify the molecular mechanisms controlling vascular pattering in woody plants and explain the link(s) between 
short and long distance auxin transport in species with extensive stem development.

Here we present the first comprehensive account of the PIN, $A U X / L A X$, and $A B C B$ gene families in Populus, which contain 16, 8 , and 20 members respectively. We investigate the history of gene family members relative to each other within Populus and relative to proposed orthologs in Arabidopsis. Through phylogenetic analysis we describe the timing of the diversification of the PIN, $A U X / L A X$, and $A B C B$ gene families relative to when plants colonized land. Because the transport function of the $A B C B$ proteins is less understood and their specificity for auxin has not been completely elucidated, we model the protein structures for Populus ABCBs and compare these to known Arabidopsis ABCB transporters. We then provide expression data for all putative auxin transporters in Populus, including presence or absence data for each gene in the cortex, phloem, cambial zone, and xylem of mature stems. We present quantitative RT-PCR expression levels for whole plantlets, internodes just beginning to form secondary vascular tissue, roots and developing xylem from mature stems. Lastly, in order to determine the most likely contributors to the positive feedback mechanism driving "canalization" of auxin flow during vascular development, we test the response of $P I N, A B C B$, and $A U X / L A X$ genes to exogenous IAA application. These findings should lay the foundation for the functional characterization of members of each family and suggest which proteins are likely to be important regulators of secondary growth.

\section{MATERIALS AND METHODS PLANT MATERIAL}

Populus tremula $\times$ alba hybrid clone INRA 717-1B4 was chosen for all experimental procedures. In vitro plants were grown on half-strength Murashige and Skoog (MS) supplemented with $2 \%$ sucrose, $0.25 \mathrm{mg} \mathrm{ml}^{-1} \mathrm{MES}, 0.04 \mathrm{mg} \mathrm{ml}^{-1}$ glycine, and $0.2 \mathrm{mg} \mathrm{ml}^{-1}$ myo-inositol at $25 \pm 2{ }^{\circ} \mathrm{C}$ under $16 \mathrm{~h}$ day length conditions using GE 20W F20T12 growth lamps. Greenhouse plants were grown in 2:1:1 promix HP: perlite:vermiculite supplemented with 19-6-12 N-P-K slow release fertilizer. Greenhouse temperatures were maintained around $22 \pm 5^{\circ} \mathrm{C}$ and day light supplemented to achieve a $16 \mathrm{~h}$ day length using metal halide lamps.

\section{IDENTIFICATION OF PIN, AUX/LAX, AND ABCB GENE AND PROTEIN FAMILIES}

Populus trichocarpa gene and protein sequences were retrieved from the Joint Genome Institute's (JGI) P. trichocarpa v.1.1 database $^{1}$. Henceforth we refer to these genes and gene families as PtrPIN, PtrAUX, and PtrABCB. When reporting expression data, we will refer to the same genes from $P$. tremula $\times$ alba (abbreviated as Pta, i.e., PtaPIN1). The PIN and AUX/LAX sequences had been previously annotated and we maintained the original nomenclature including the $A U X$ and $L A X$ names for every member of the $A U X / L A X$ family from $P$. trichocarpa (i.e., PtrAUX1-LAX5). Every sequence was used as query with the BLASTn algorithm to search the National Centre for Biotechnology Information (NCBI)

\footnotetext{
${ }^{1}$ http://genome.jgi-psf.org/Poptr1_1/Poptr1_1.home.html
}

nucleotide collection database to confirm sequence identity. Putative $A B C B$ genes in the $P$. trichocarpa genome were identified in the same database using 22 Arabidopsis $A B C B$ gene sequences retrieved from the Arabidopsis Genome Initiative Research database $(\text { TAIR })^{2}$. The JGI P. trichocarpa v.1.1 database was also searched using the terms "MDR" and "ATP" as queries. A third search was conducted using the retrieved sequences to interrogate the Populus DataBase (PopulusDB) ${ }^{3}$. Finally all retrieved sequences were confirmed as encoding putative auxin transporters by searching the phytozome v.7.0 database ${ }^{4}$. All the remaining PIN, $A U X / L A X$ and $A B C B$ sequences from other species were retrieved from phytozome v.7.0, TAIR10, The Rice Genome Annotation Project $^{5}$, and MaizeGDB ${ }^{6}$. The complete list of retrieved genes is provided in Table A4 in Appendix. All sequences were inspected for redundancy and presence of pseudogenes and invalid gene models were discarded. ABCB protein sequences were used as queries to search the PROSITE database ${ }^{7}$ to confirm the presence of the TMD-NBD-TMD-NBD (transmembrane domain, nucleotide-binding domain) structure and the $\mathrm{ABC}$ C-motif. This allowed to rule out the presence of $\mathrm{ABC}$ half transporters and other ABC proteins not belonging to class B (Sanchez-Fernandez et al., 2001) and to classify the genes according to their full length structure, conserved motifs, sequence similarity, and EST support. Intron-exon structures of $P$. trichocarpa PIN, $A U X / L A X$, and $A B C B$ genes were produced using the online tool GSDS, Gene Structure Display Server (Guo et al., 2007) ${ }^{8}$. The genome representation for Populus was created using the online tool SyMAP v. $3.5^{9}$

\section{PtrABCB, PIN, AND AUX/LAX STRUCTURE ANALYSIS AND PtrABCB MODELING}

Transmembrane domains were predicted using the online tools TMHMM Server v.2.0 ${ }^{10}$ and Aramemnon ${ }^{11}$. The protein structure of Sav1866 and MDR1 were obtained from the PDB (Protein Data Bank) database ${ }^{12}$. The predicted protein structures of AtABCB1 and 4 have been previously generated by Yang and Murphy (2009). Arabidopsis templates (ABCB1 or 4) were chosen based on closest sequence identity. To generate the alignment files of Populus ABCB protein sequences and Arabidopsis ABCB sequences, Multialin ${ }^{13}$ was used with default settings. The output file was manually edited to meet Modeller 9v5 requirements ${ }^{14}$. The predicted 3D protein structure was generated using the python script Modeller 9v5. Three structures were generated and the quality was determined according to the manual (Wiederstein and Sippl, 2007). The best model was used for substrate docking. Furthermore, the

\footnotetext{
2 www.arabidopsis.org

${ }^{3}$ http://www.populus.db.umu.se

${ }^{4}$ http://www.phytozome.org

${ }^{5}$ http://rice.plantbiology.msu.edu/

${ }^{6}$ http://www.maizegdb.org/

${ }^{7}$ http://ca.expasy.org/prosite/

${ }^{8} \mathrm{http}: / / g s d s . c b i . p k u . e d u . c n / i n d e x . p h p$

${ }^{9}$ http://www.symapdb.org/.

${ }^{10} \mathrm{http}: / /$ www.cbs.dtu.dk/services/TMHMM/

$11 \mathrm{http}$ ///aramemnon.uni-koeln.de/

12 http://www.rcsb.org/pdb/home/home.do

$13 \mathrm{http}: / /$ bioinfo.genotoul.fr/multalin/multalin.html

$14 \mathrm{http} / / /$ salilab.org/modeller/release.html
} 
quality of the protein model was tested using the program ProSA ${ }^{15}$. Substrate docking was performed using MEDOCK ${ }^{16}$. PDB files of all proteins were translated into pdbq files using the PDB2PQR server ${ }^{17}$. For substrate docking prediction, the nucleotide-binding folds (NBFs) were removed. All loops connecting the TMDs were removed to reduce the size of the file. Finally, the pdbq file of IAA was produced with the Dundee PRODRG2 Server (Dolinsky et al., 2004, 2007 $)^{18}$. Each run had a docking repeat of five times and four runs were performed, resulting in a total of 20 molecules docked to the protein structure. Protein models were displayed using $\mathrm{PyMol}^{19}$.

\section{PHYLOGENETIC ANALYSIS}

Phylogenic reconstruction was conducted using the coding sequences of 18 species, including 3 monocotyledonous and 10 dicotyledonous plants. Sequences from the green algae Chlamydomonas reinhardtii (Merchant et al., 2007) and Volvox carteri (Prochnik et al., 2010), the moss Physcomitrella patens (Rensing et al., 2008) and the lycopod Selaginella moellendorffii (Banks et al., 2011) were also included. For each coding sequence, three types of trees were retrieved from two different alignments. The first alignment was generated in concert with the tree search, a method called "dynamic homology" (Wheeler, 1996). 149, 68, and 245 unaligned coding sequences from the PIN, AUX/LAX, and $A B C B$ families (Table A4 in Appendix) were read into the phylogenetic program POY v.4.1.2 (Varón et al., 2009) and trees and alignments were searched simultaneously for the least costly sequence alignment and tree topology combination under the parsimony criterion. A second alignment was generated in the program MAFFT (Katoh et al., 2009), where the same sequences were aligned under a gap opening cost of 4 and a gap extension cost of 0.05 . This alignment was then input to the program Gblocks v.0.91b (Castresana, 2000; Talavera and Castresana, 2007), which removes regions with multiple gaps and of dubious homology. Gblocks was run with default settings, except that gaps were allowed in all parts of the resulting alignment (such as in cases where one or a few sequences have a clear insertion or deletion). The alignment output by Gblocks was then used for tree searching in POY, where it was read as pre-aligned. Both unaligned and aligned POY tree searches were immediately followed by bootstrap searches, where 100 pseudoreplicates were searched starting with one Wagner tree each. Tree searches were conducted on a parallel computing cluster, using 24 processors searching for a maximum of $6 \mathrm{~h}$ of automated searching (in which POY decides on the best combination of builds, swapping, ratchet, and fusing) with dynamic homology and 16 processors for the pre-aligned data. For dynamic homology, in both the tree searches and the bootstrap calculations, the data were divided by the program into seemingly homologous blocks before searching using the command "auto_sequence_partition," which greatly increases search speed. For all POY searches, the costs of

\footnotetext{
${ }^{15} \mathrm{http}: / /$ www.came.sbg.ac.at/typo3/index.php?id = prosa

$16 \mathrm{http}: / /$ medock.csbb.ntu.edu.tw

${ }^{17} \mathrm{http}: / /$ pdb2pqr.sourceforge.net

${ }^{18} \mathrm{http}: / /$ davapcl.bioch.dundee.ac.uk/prodrg,

${ }^{19} \mathrm{http}: / /$ pymol.sourceforge.net
}

transitions, transversions, and insertion/deletion events were the same.

The alignment from Gblocks was also used for a maximum likelihood search in RaxML (Stamatakis et al., 2008) on the CIPRES Science Gateway (Miller et al., 2010) ${ }^{20}$. The alignment was first uploaded and converted to relaxed Phylip format and then tree searches were performed with likelihood bootstrap in which the best tree is reported along with the results of a 100-pseudoreplicate bootstrap calculation. The program was allowed to determine the best model (the GAMMA Model was chosen) and other parameters automatically before tree searching. All trees were visualized and edited using FigTree v.1.3.1 ${ }^{21}$

\section{DNA AND RNA ISOLATION AND cDNA SYNTHESIS}

Total RNA from whole in vitro-grown plantlets, internodes, roots, and developing xylem was extracted using the Spectrum Plant Total RNA Kit (Sigma-Aldrich, St. Louis, MO, USA) according to manufacturer's instructions. Aliquots of approximately $100 \mathrm{mg}$ developing xylem tissue were homogenized with a Mini Bead Beater (BioSpec Products Inc., Bartlesville, OK, USA) and stainless steel beads. mRNA from $20 \mu \mathrm{m}$-thick frozen sections from the cortex, secondary phloem, cambium, and secondary xylem was extracted using the DynaBeads mRNA Direct Kit (Invitrogen, Carlsbad, CA, USA) according to manufacturer's instructions. DNA was extracted using the DNeasy Plant Mini Kit (Qiagen, Valencia, CA, USA) according to manufacturer's instructions using approximately $100 \mathrm{mg}$ fresh leaf tissue. DNA and RNA concentrations were measured with a NanoDrop $2000^{\mathrm{TM}}$ (Thermo Scientific, Waltham, MA, USA). Total RNA was treated with TURBO DNAfree $^{\mathrm{TM}}$ (Ambion, Austin, TX, USA) according to manufacturer's instructions. cDNA was synthesized from $1.5 \mu \mathrm{g}$ of total RNA using SuperscriptII reverse transcriptase (Invitrogen, Carlsbad, CA, USA) with the oligodt 20 primer. RT-PCR reaction cycles were carried out according to manufacturer's instructions including a final 20 min incubation step with RNAseH (Invitrogen, Carlsbad, CA, USA). cDNA concentration was measured with a Nanodrop

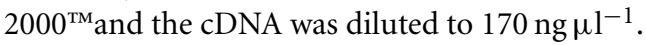

\section{AMPLIFICATION, CLONING AND SEQUENCING OF 3' END PCR PRODUCTS}

In order to amplify the $3^{\prime}$ end untranslated region (UTR) of transcripts that could not be detected in quantitative real time PCR (qRT-PCR) reactions with at least three different primer pairs, reverse transcription reactions were carried out using the Adp1-dt17 primer (Kramer et al., 1998) and SuperscriptII reverse transcriptase according to manufacturer's instructions. cDNA was amplified using the Adp1 primer coupled to the corresponding forward primer specifically designed to amplify the $3^{\prime}$ end of the transcript (the complete list of primers is provided in Table A5 in Appendix). The PCR amplifications were carried out with Taq DNA polymerase (SIGMA, St. Louis, MO, USA) or Amplitaq ${ }^{\oplus}$ Gold DNA polymerase (Applied Biosystems ${ }^{\mathrm{TM}}$, Foster City, CA, USA) according to manufacturer's instructions. PCR

\footnotetext{
${ }^{20} \mathrm{http} / / /$ www.phylo.org/news/raxml.php

${ }^{21} \mathrm{http}: / /$ tree.bio.ed.ac.uk/software/figtree/.
} 
products were run on $1 \%$ agarose gels, gel purified using the Zymoclean ${ }^{\mathrm{TM}}$ Gel DNA Recovery Kit (Zymo Research, Irvine, CA, USA) and cloned into the $p G E M^{\circledR}$-T Easy Vector Systems (Promega, Madison, WI, USA). Colonies were grown on LB plates containing $100 \mathrm{mg} / \mathrm{ml}$ ampicillin. Following PCR amplification, positive colonies were grown in $4 \mathrm{ml}$ of LB medium containing $100 \mathrm{mg} / \mathrm{ml}$ ampicillin, at $37^{\circ} \mathrm{C}$, over night. Plasmid DNA was extracted using the Qiagen Plasmid Mini Kit (Qiagen, Valencia, CA, USA) according to manufacturer's instructions. Plasmids were sequenced by Eurofins MWG Operon (Huntsville, AL, USA). Sequences were aligned using the Vector NTI Advance ${ }^{\mathrm{TM}}$ 10.3.0 AlignX module (Invitrogen, Carlsbad, CA, USA).

\section{QUANTITATIVE RT-PCR}

Quantitative real time PCR was carried out on the MX3000P and MX3005P systems (Stratagene, La Jolla, CA, USA) using Brilliant $^{\mathrm{TM} S Y B R}{ }^{\circledR}$ Green QPCR Master Mix (Stratagene, La Jolla, CA, USA) according to manufacturer's instructions. The SYBR ${ }^{\circledR}$ Green (with dissociation curve) experimental setup was used. Plates were manually loaded and reactions were carried out in a total volume of $20 \mu \mathrm{l}$, using $75 \mathrm{ng}$ of cDNA per reaction. Reactions were run in triplicate. Primer pairs were designed using Primer3 software ${ }^{22}$, analyzed with OlygoAnalyzer 3.1 software $^{23}$ for melting temperature, oligo-, hetero-dimer, and hairpin structure formation, synthesized by Integrated DNA Technologies (IDT, IA) and tested with conventional PCR to verify amplification of a single product. Following primer titration, a final concentration of $250 \mathrm{nM}$ for each primer was chosen. In qRT-PCR experiments the following thermal cycling conditions were used: activation step of $10 \mathrm{~min}$ at $95^{\circ} \mathrm{C} ; 40 \mathrm{cycles}$ of $30 \mathrm{~s}$ at $95^{\circ} \mathrm{C}, 25 \mathrm{~s}$ at $57^{\circ} \mathrm{C}, 25 \mathrm{~s}$ at $72^{\circ} \mathrm{C}$; fluorescence was collected at the end of each extension step. A melting curve analysis was performed.

Efficiency-corrected expression values were calculated based on standard curves for all genes (Livak and Schmittgen, 2001; Pfaffl, 2001). Standard curves were run in triplicate for every gene in every cDNA batch and amplification efficiencies were calculated from the standard curve slopes. Baseline-subtracted and ROX-normalized fluorescence readings were collected with the MX3005P software v.4.01. Expression values were normalized to the geometric mean of four housekeeping genes (PtaPD-E1, PtaUBQ1, PtaTUA2, PtaACT2) that were found, in our hands, to have the highest amplification efficiency and most stable expression across different tissues (Vandesompele et al., 2002; Brunner et al., 2004; Gutierrez et al., 2008). For expression following exogenous IAA application, the same set of normalizers was used in a comparative quantitation experiment comparing treated and untreated control tissues.

\section{IAA TREATMENTS}

Two-month-old P. tremula $\times$ alba was grown in the greenhouse. Approximately 1-cm-long segments of internodes between four and eight nodes beneath the shoot apex and actively growing root tips were collected and incubated at room temperature in $30 \mu \mathrm{M}$ IAA in liquid growth media (half-strength MS salts, $2 \%$ sucrose,

\footnotetext{
${ }^{22} \mathrm{http}: / /$ frodo.wi.mit.edu/primer3

${ }^{23} \mathrm{http}: / /$ www.idtdna.com/analyzer/Applications/OligoAnalyzer
}

$0.25 \mathrm{mg} \mathrm{ml}^{-1} \mathrm{MES}, 0.04 \mathrm{mg} / \mathrm{ml}$ glycine, and $0.2 \mathrm{mg} \mathrm{ml}^{-1}$ myoinositol) for $6 \mathrm{~h}$ in the dark following a $15 \mathrm{~min}$ vacuum infiltration. The same conditions were used for negative controls (no IAA). Tissues were frozen in liquid $\mathrm{N}_{2}$ and ground for RNA extraction.

\section{RESULTS}

\section{CHROMOSOMAL DISTRIBUTION AND GENE DUPLICATION IN THE PIN, AUX/LAX, AND ABCB FAMILIES OF POPULUS}

Nearly every locus coding for a PIN, AUX/LAX, or ABCB protein has a corresponding paralogous locus in another chromosomal block (Figure 1). Populus has exactly twice the number of PIN (16) and AUX/LAX (8) genes as Arabidopsis (eight and four, respectively) and these genes form pairs with highly similar coding sequences, which may be the consequence of the relatively recent genome duplication (Figures 1, 2, and 3). Neither the PIN loci nor the $A U X / L A X$ loci appear to be derived from tandem duplications. In contrast, three tandem duplicated $A B C B$ loci pairs (PtrABCB2-PtrABCB8, PtrABCB10-PtrABCB11, and PtrABCB13-PtrABCB14) are present in the Populus genome. Unlike the PIN and $A U X / L A X$ families, the $A B C B$ genes are more randomly distributed between corresponding and noncorresponding duplicated regions, with nine members that do not present any paired gene on another chromosome (Figure 1).

\section{GENE AND PROTEIN STRUCTURE OF THE PIN, AUX/LAX, AND ABCB FAMILIES OF POPULUS}

We identified a total of 44 Populus genes encoding putative auxin transport proteins, including 16 PIN, 8 AUX/LAX, and 20 PtrA$B C B$ loci. The complete list of $P$. trichocarpa PIN, AUX/LAX, and $A B C B$ gene names, gene models, and loci can be found in Table A2 in Appendix. The PIN genes of Populus present a conserved intron-exon organization which is illustrated in Figure A1 in Appendix. The same structural characteristics are present across PINs from different plant species including Arabidopsis (Mravec et al., 2009; Wang et al., 2009; Shen et al., 2010). The proteins belonging to the PtrPIN family range from 347 to 650 amino acids in length. In Populus, seven, three, and six PIN proteins present long, reduced and short central hydrophilic domains respectively. In general, there is no strict correlation between the length of the genomic sequence of loci coding for auxin transporters and their protein product length (Figure A1 and Table A3 in Appendix). One locus (PtrPIN14) is classified as encoding a pseudogene. The proteins for the PtrAUX/LAX family range from 465 to 492 amino acids and present the most conserved sequence among the three families of putative auxin transporters. Their primary sequence is generally conserved across the plant kingdom and Populus has twice the number of $A U X / L A X$ coding loci compared to Arabidopsis. All of the PtrAUX/LAX proteins have 11 predicted transmembrane domains. All the $A B C B$ loci from $P$. trichocarpa encode proteins with a repeated TMD-NBD structure and carry a predicted nucleotide-binding domain signature ([AG]- $\times(4)-G-$ K-[ST]; Rea, 2007; Verrier et al., 2008). Their length varies between 1141 and 1578 amino acids and the two regions integral to the plasma membrane are highly hydrophobic and comprise 7-12 transmembrane helices. In addition to these two conserved modules, a more variable and less hydrophobic linker region connects the first NBD to the second TMD in all PtrABCB proteins. 


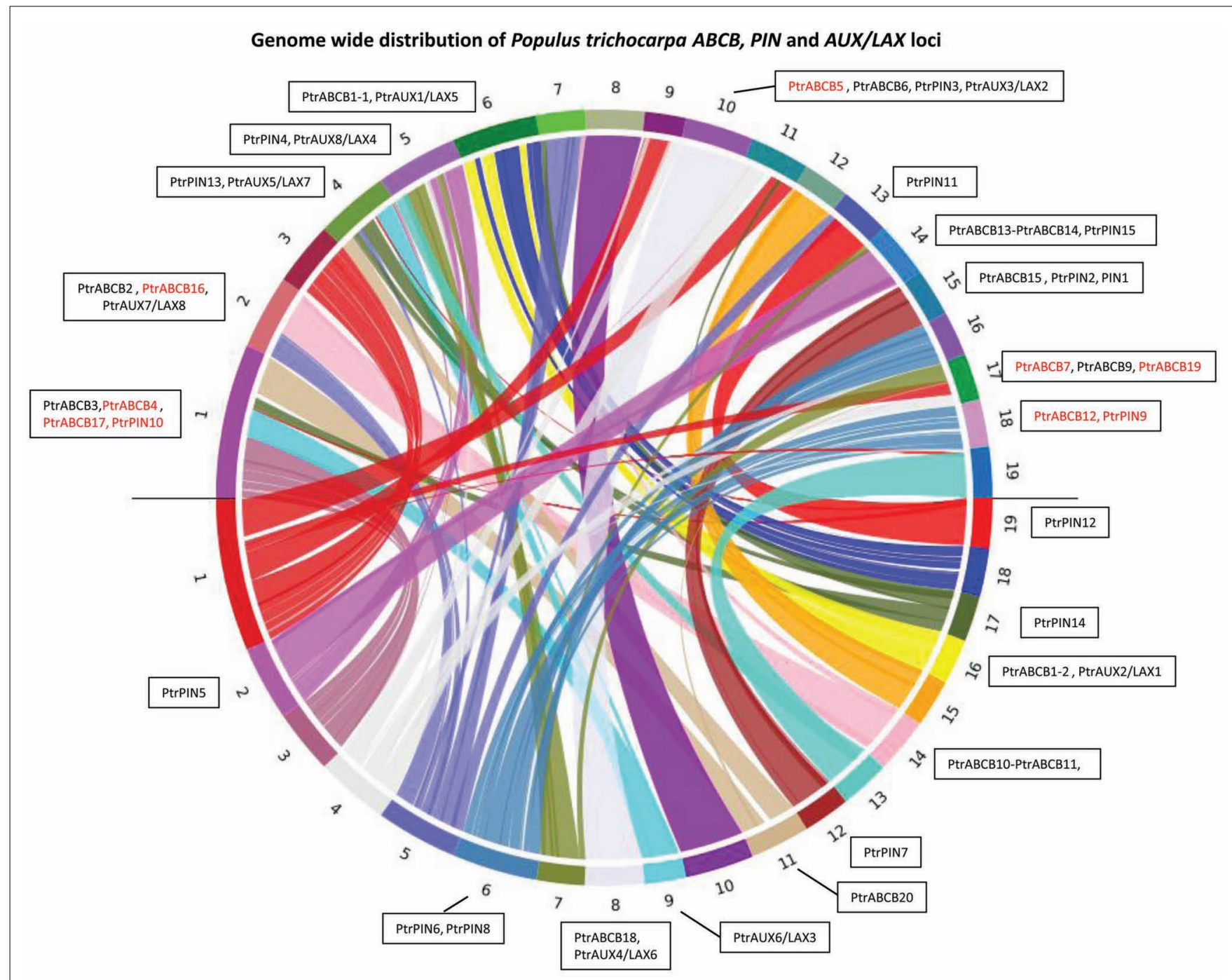

FIGURE 1 | Chromosome distribution of PtrPIN, PtrAUX/LAX, and PtrABCB genes. The online tool symap v.3.5 was used to blast the Populus trichocarpa genome against itself and find duplicated regions. Populus has 19 chromosomes in the haploid state, shown here mapped onto a circle with homologous pairs along the upper and lower semi-circumferences. The color coded ribbons link one region with the correspondent homologous chromosomal segments. All PIN, AUX/LAX, and $A B C B$ genes are assigned to a chromosome based on their map position. Red coded genes do not have any unique match on another locus in the genome. For a detailed list of these genes, see Table A2 in Appendix.

\section{IDENTIFICATION OF PREDICTED IAA MEMBRANE TRANSPORTERS FROM THE ABCB FAMILY OF POPULUS}

After analysis of the primary structure of the PtrABCB proteins, models of tertiary structure were produced using all 20 $\mathrm{ABCB}$ amino acid sequences. Structural models were displayed using PyMol (Figure A2 in Appendix) in order to determine which PtrABCBs are the most likely candidates for IAA transport. Although pairwise comparison of amino acid sequences can provide a first estimate of which proteins are the true orthologs of confirmed Arabidopsis auxin transporters (AtABCB1, AtABCB19, and AtABCB4), this information should be supported with the identification of IAA docking sites and transmembrane barrel structure predictions (Yang and Murphy, 2009). Among all PtrA$\mathrm{BCBs}, 10$ are predicted to have one or more IAA binding sites (Figure A2 in Appendix). In Arabidopsis, IAA is primarily docked at two binding sites in the TMDs of ABCB19 while ABCB4 has a unique additional binding site (Yang and Murphy, 2009). In Populus, ABCB1.1/ABCB1.2 and ABCB19 have the most similar sequence to AtABCB1 and AtABCB19 and have two, five, and three predicted binding pockets respectively.

\section{RECONSTRUCTION OF THE PHYLOGENETIC RELATIONSHIPS IN THE PIN, AUX/LAX, AND ABCB GENE FAMILIES OF POPULUS}

All three phylogenetic analyses (parsimony using unaligned and aligned sequences and maximum likelihood with aligned sequences) generally resulted in well resolved, reasonable, highly supported trees, indicating considerable phylogenetic signal in the sequence data, which was robust to different methods of analysis. Here we show the trees for all three gene families found under maximum likelihood and the tree found under dynamic homology 


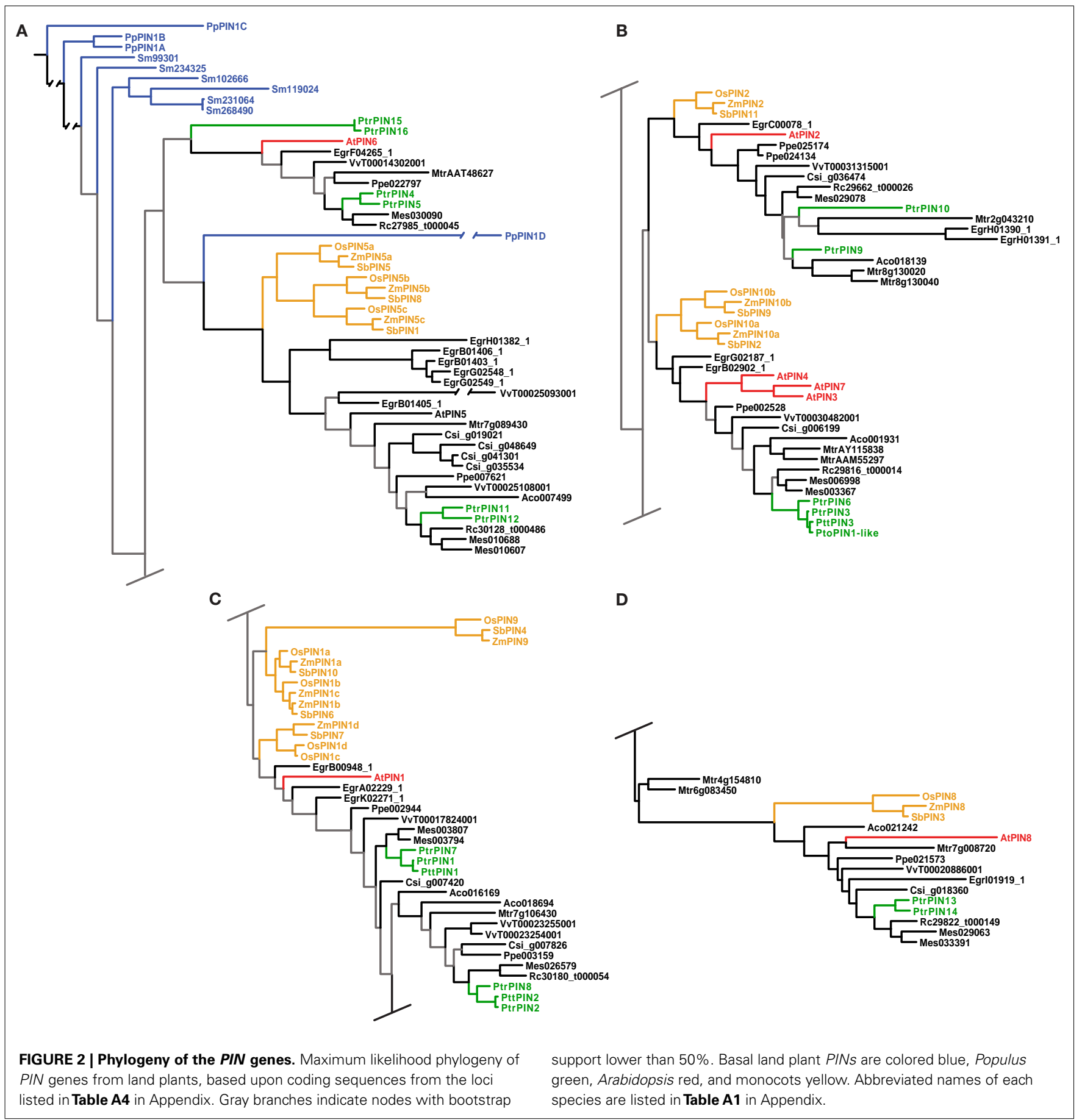

and parsimony for the ABCB family (Figures 2, 3, and 4; Figure A3 in Appendix). The three different analyses showed the same general patterns in each gene family, although the PIN analysis was more sensitive to the difference between likelihood and parsimony, the latter producing long, pectinate clades containing a mixture of taxonomic groups.

The PIN genes of basal land plants (Physcomitrella and Selaginella in our analysis) cluster at the base of the tree, with the exception of PpPIN1D (Figure 2A). The placement of $P$ PPIN1D may indicate an erroneous or highly derived sequence, as its placement was unstable and with low bootstrap support and it was recovered in the likelihood tree on an extremely long branch. The angiosperm PINs initially split into two large clades, with subsequent splits that show the monocot/dicot divergence four or five times, although support for several of these nodes is weak (Figure 2). There is also the frequent occurrence of clear sister pairs of PINs in Populus. 


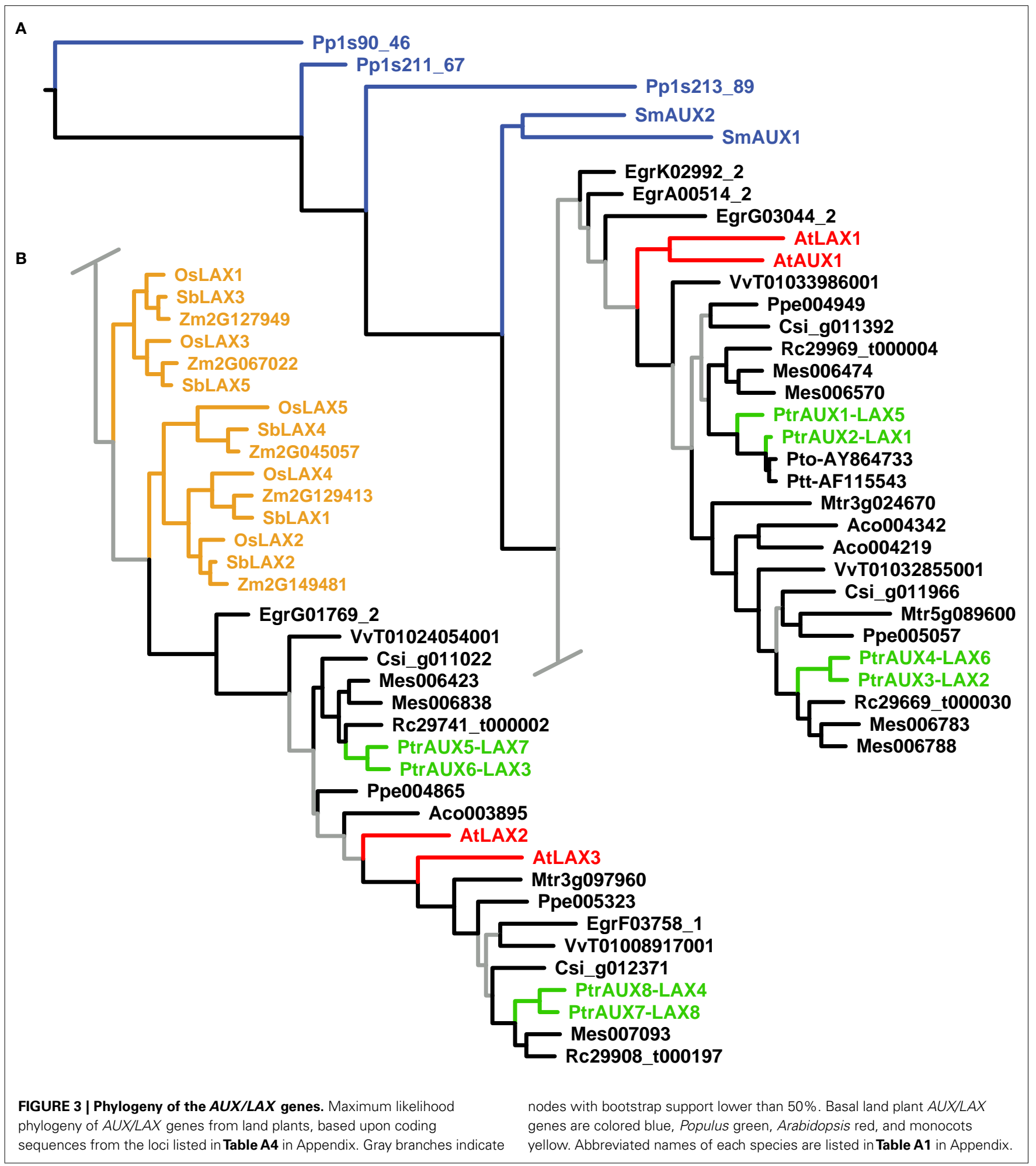

The $A U X / L A X$ analysis similarly places the basal land plant $A U X / L A X$ genes in a grade at the base of the tree followed by two large clades of angiosperms (albeit with weak support; Figure 3). The monocot $A U X / L A X$ genes were recovered as two closely related clades under maximum likelihood (Figure 3B) but were recovered as a single clade when the aligned data were analyzed under parsimony (trees not shown). All Populus AUX/LAX genes were recovered as sister pairs or, in the case of PtrAUX1-LAX5 and PtrAUX2-LAX1, as closely related in a clade with the $P$. tomentosa and P. tremula $\times$ tremuloides AUX/LAXs. 


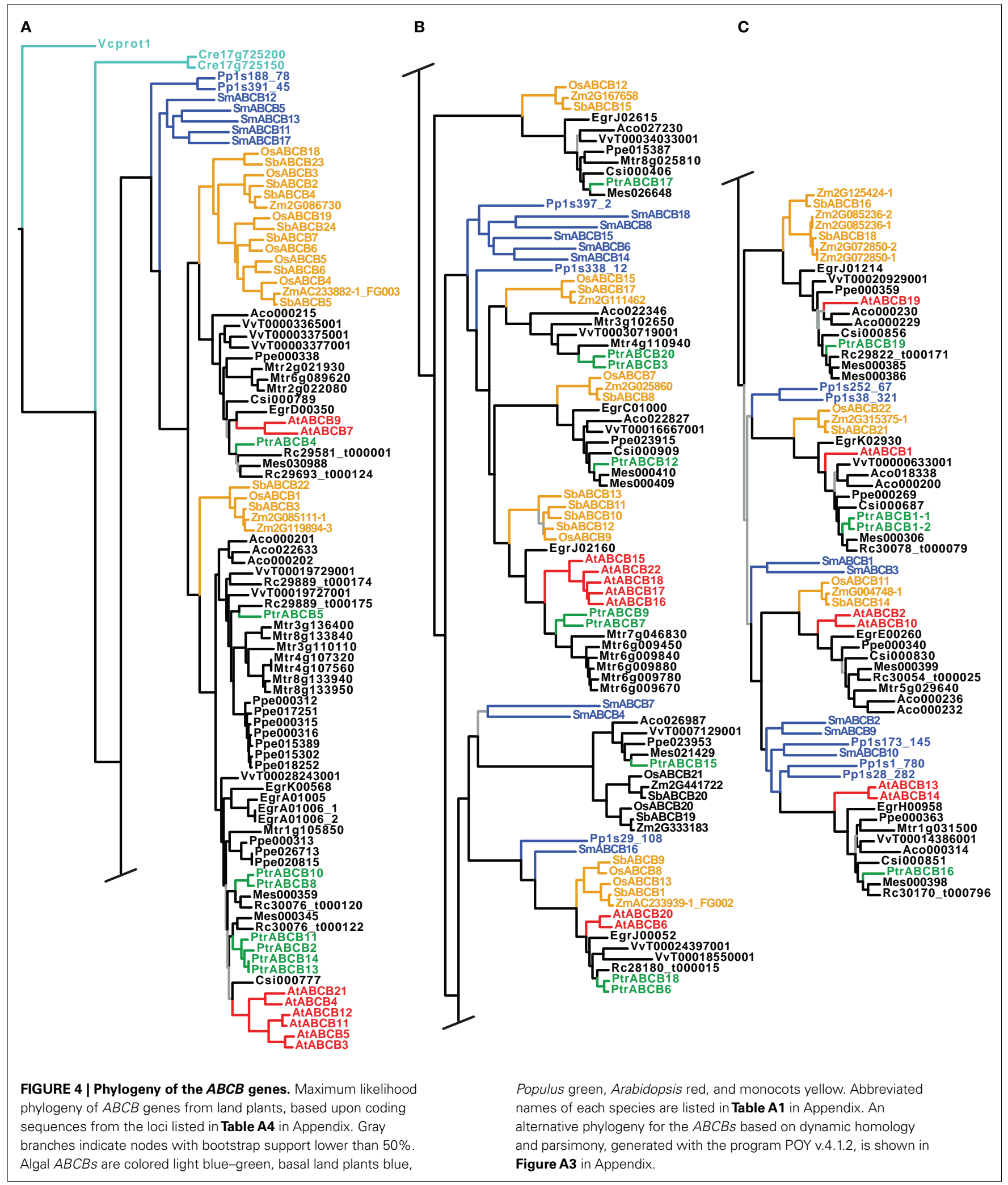

In contrast to the PIN and AUX/LAX trees, clades, or paraphyletic grades of basal land plant $A B C B$ s were recovered in several different locations throughout each tree, often as sister to angiosperm clades that subsequently showed the monocot/dicot split (Figure 4). We included coding sequences from the green algae in our $A B C B$ analysis: two putative $A B C B$ transporters 
from C. reinhardtii (Cre17_g725200 and Cre17_g725150) and one $A B C B$-like sequence from $V$. carteri (Vcprot1), the latter used to root each $A B C B$ tree. The inclusion of the algal sequences and the use of Volvox as a root appear valid, as they are not recovered on especially long branches, and Physcomitrella and Selaginella are appropriately placed on the first branches of each tree. In the maximum likelihood tree, we recovered 10 separate clades of monocot $A B C B$ s, as well as an apparent expansion of the $A B C B$ s in several angiosperm species, including Medicago truncatula and Prunus persica (Figures 4A,B). Among the Populus $A B C B$ s, only few were recovered in clear sister pairs. The tree found under dynamic homology for the $A B C B s$ recovered almost identical groupings of basal land plant, monocot, and dicot $A B C B s$ as those trees found using aligned sequences, but the relationships among these clades or groups differed. For example, a clade containing $O s A B C B 12$ and Mes026648 (top of Figure 4B) was recovered as a paraphyletic grade immediately after the algal sequences in the dynamic homology tree (Figure A3A in Appendix).

\section{TISSUE-SPECIFIC AND IAA-INDUCED EXPRESSION OF PtaPINs PtaAUX/LAXs, AND PtaABCBs}

Expression of all PIN, $A U X / L A X$, and $A B C B$ gene family members in $P$. tremula $\times$ alba was characterized for whole plantlets, roots, and stem tissues from several developmental stages through qRT-PCR (Figures 6-8). Whole in vitro-grown plantlets that were old enough to have initiated secondary growth were used as an initial screen and showed that over half of the PtaPINs and PtaAUX/LAX genes were expressed at abovetrace levels, while only four or five PtaABCBs showed abovetrace expression. Internodes that spanned the region of secondary growth initiation in greenhouse-grown plants should reflect combined expression in several distinct tissues, including cortex, vascular cambium, developing secondary vasculature, and primary xylem parenchyma. Here PtaPIN1, 6, and PtaABCB1.1 show high expression levels, with lower levels of PtaPIN7, 11, 15, 16, and PtaABCB7 (Figures 6 and 8). Developing secondary xylem removed from beneath the bark in 6-month-old greenhouse-grown trees showed high expression of PtaPIN1 and PtaABCB1.1, with lower levels of PtaABCB7. Roots showed low expression levels of most genes, which may simply reflect the fact that the roots collected were relatively mature and composed largely of parenchyma, rather than a concentration of actively growing root tips. PtaAUX/LAX genes were expressed at relatively uniform levels across all tissues and developmental stages (Figure 7), although expression levels were highest for developing xylem, where very high levels of PtaAUX2 were detected.

In order to perform an expression screen (RT-PCR) with higher spatial resolution in developing woody stems, basal internodes approximately 100 nodes and $2.5 \mathrm{~m}$ down from the stem apex of 6-month-old Populus were freeze-sectioned and tissue collected from the cortex, secondary phloem, cambial zone (restricted to cambial initials and mother/daughter cells), and secondary xylem. Developing secondary xylem and phloem were discarded in order to obtain the most pure collections of tissues possible. Given that, the number of members of all families that are expressed in each tissue is striking (Figures 5-8). Only PtaPIN9, 10, and 12 and PtaABCB5 and 10 were not expressed in any tissue (Figures $\mathbf{6}$ and 8), and although some of the transcripts detected through RT-PCR are likely expressed at very low levels, it is clear that expression of many previously undescribed members (e.g., PtaPIN6, 7, 15, and 16 and PtaABCB1.1 and 7) is widespread in Populus stems. Also striking is the fact that several members of all three transport families are expressed in mature secondary xylem, from which all mRNA is derived from living ray parenchyma cells.

Because a positive feedback mechanism is fundamental to the canalization of auxin flow during vascular development, we also tested the auxin response of members of the PtaPIN, PtaAUX/LAX, and $P \operatorname{ta} A B C B$ gene families in roots and internodes from 2month-old plants, following exogenous IAA application, via qRTPCR. PtaPIN1, 2, and 7 and PtaAUX5 and 6 were strongly upregulated in developing internodes, with PtaPIN15 and 16 showing a more moderate increase (Figure 9). In contrast, PtaPIN3 and 8 were strongly upregulated in roots, with PtaAUX6 and PtaABCB7 showing a lower expression level.

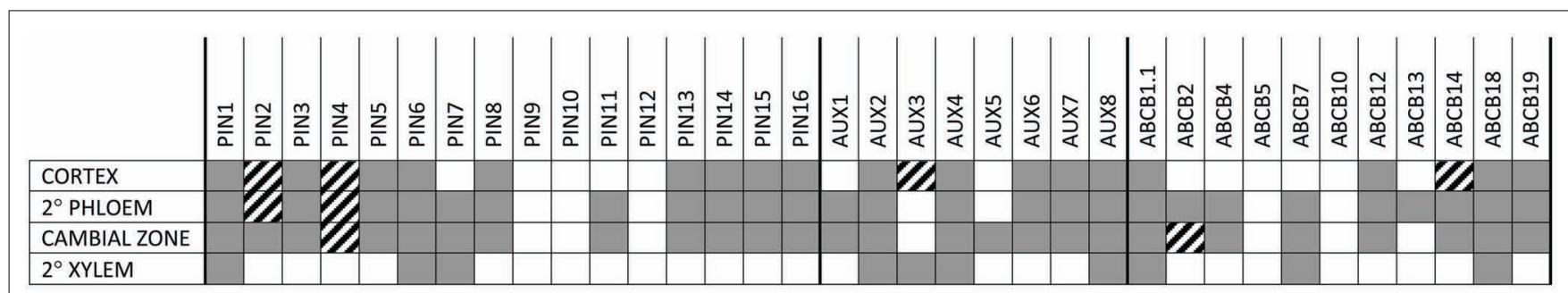

PRESENT

VARIABLE

ABSENT

FIGURE 5 | Analysis of tissue-specific expression of PIN, AUX/LAX, and $\boldsymbol{A B C B}$ transcripts. Presence or absence of transcripts of genes coding for putative auxin transport proteins in the cortex, secondary phloem, cambial zone (i.e., initials and mother/daughter cells), and mature secondary xylem of Populus tremula $\times$ alba as determined by RT-PCR. Consensus of four biological replicates is shown, where GRAY = PRESENT, WHITE =ABSENT, and CROSS-HATCHED =VARIABLE among biological replicates. Samples were taken from the base of 6-month-old trees during active growth, approximately 100 internodes down from the top of the tree at a diameter of about $2 \mathrm{~cm}$. 

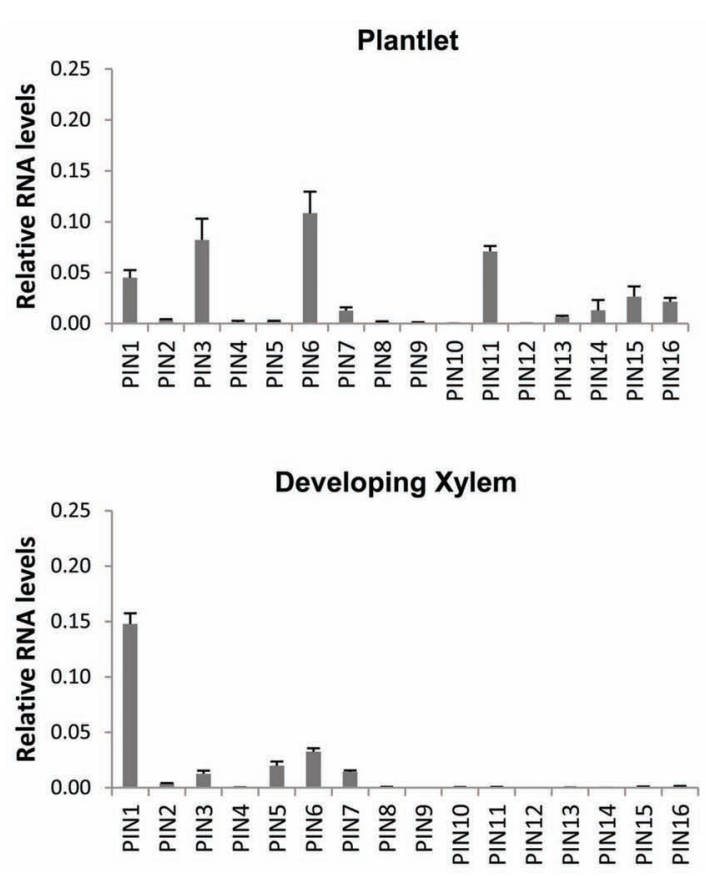

FIGURE 6 | Quantification of PIN transcripts expression by qRT-PCR. PIN genes show tissue-specific expression profiles that may reflect a role in directional auxin transport in developing vasculature, with PtaPIN1 highly expressed across all tissues. PtaPIN6, 7, 15, and 16 were expressed in internodes and have not been described before. Total RNA was extracted from four biological replicates and qRT-PCR standard curves and assays were run in triplicate. Expression values were
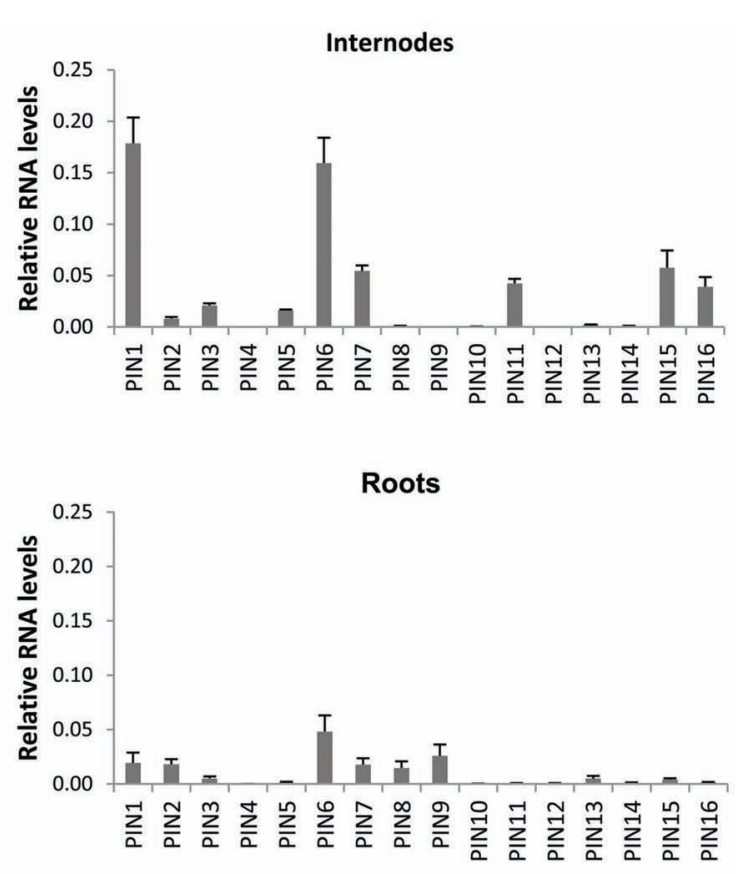

calculated via the $2^{-\Delta \Delta C t}$ method (Livak and Schmittgen, 2001; Pfaffl 2001) and baseline-corrected fluorescence values were normalized against the geometric mean of PtaPD-E1, PtaTUA2, PtaUBQ, PtaACT2. These reference genes were stably expressed across all tissues with the exception of developing xylem; this means that it is permissible to compare expression levels within any single tissue as well as across whole plantlets, internodes, and roots. Error bars represent the SEM.

\section{DISCUSSION}

\section{THE ARRAY OF PUTATIVE AUXIN TRANSPORTERS IN POPULUS REFLECTS BOTH PRE-EXISTING DIVERSITY AND EXPANSION DUE TO GENOMIC AND SEGMENTAL DUPLICATIONS}

There are twice as many members of the PIN and AUX/LAX gene families in Populus as there are in Arabidopsis and both families show a number of clear pairs based on coding sequence (e.g., PtrPIN4/5, PtrAUX3/4; Figures 2 and 3). With no clear evidence for any tandem duplication in the PIN and AUX/LAX gene families, it is possible that all gene copies were retained following the "salicoid" genome duplication (Tuskan et al., 2006). Although the functional role of these proteins has not been demonstrated in Populus, given the conserved protein structure and known specificity for IAA for most PINs in Arabidopsis (and to a lesser extent, AUX/LAX proteins), it seems likely that they have retained a function in auxin transport. To what extent new PINs have developed specialized roles in PAT in Populus is not known and the added redundancy for such an important developmental mechanism may be beneficial enough to warrant retention. Indeed, redundancy in Arabidopsis allows single PIN mutants to complete embryogenesis, whereas quadruple mutants are required before severe defects are observed (Benková et al., 2003; Friml et al., 2003). At the same time it is interesting to note that there are clear differences in expression among presumed paralogs. For instance, PtaPIN1 is expressed at much higher levels than PtaPIN7 in internodes and developing xylem. Predictions about PIN function in Populus may also be informed by structural comparisons with Arabidopsis. The "long" PINs in Arabidopsis are localized to the plasma membrane and function in PAT, whereas those with shorter structure are found in the ER (Mravec et al., 2009; Friml and Jones, 2010). PtrPIN1-3 and PtrPIN6-9 are all classified as "long" PINs (Table A3 in Appendix), but it is not known whether similar localization patterns exist in Populus.

In contrast to the PIN and AUX/LAX gene families, the number of $A B C B$ s in Populus is not expanded relative to Arabidopsis (both species include about 20 members; Table A2 in Appendix) and only a few appear as closely related gene pairs. This is perhaps not surprising given that this gene family has a much deeper history and that $\mathrm{ABCB}$ proteins transport a number of substrates in addition to IAA. There also appears to be expansion in a number of angiosperms included in our phylogeny, such as Z. mays, M. truncatula, P. persica, and Arabidopsis (Figure 4). Although there has been retention of $A B C B$ copies from both tandem duplication and whole genome duplication events in Populus, there also appears to have been loss. Much functional work is needed on Populus ABCB genes and proteins before any role in PAT can be ascribed.

\section{CANDIDATE ABCBS FOR IAA TRANSPORT FUNCTION IN POPULUS ARE SUGGESTED BY PHYLOGENETIC PLACEMENT AND PROTEIN STRUCTURE PREDICTION}

ATP-binding cassette proteins constitute a very large superfamily that has representatives across the bacteria, plant, and animal 

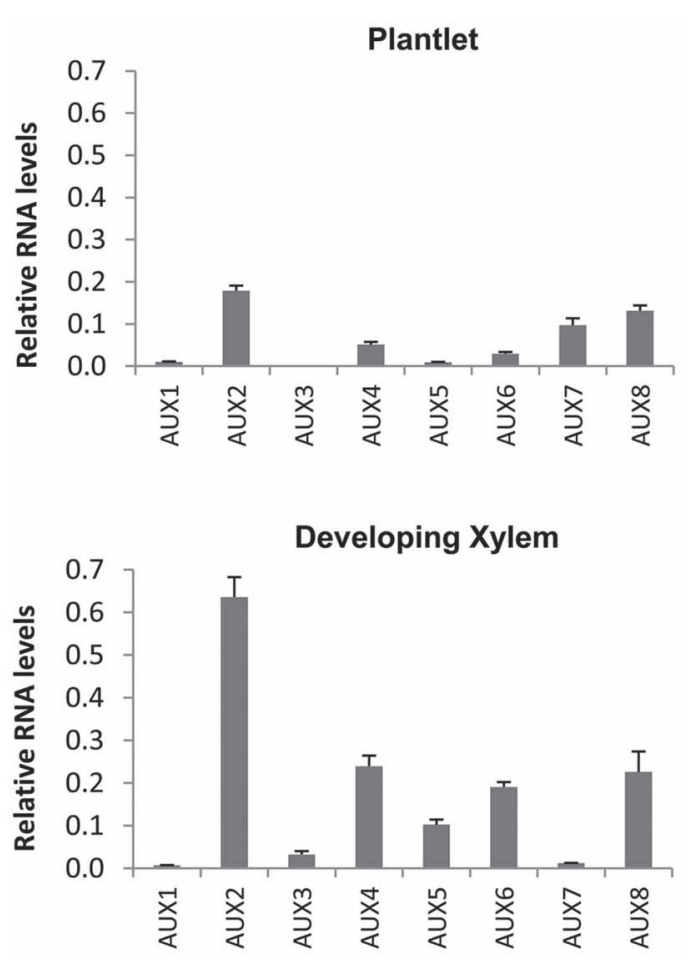
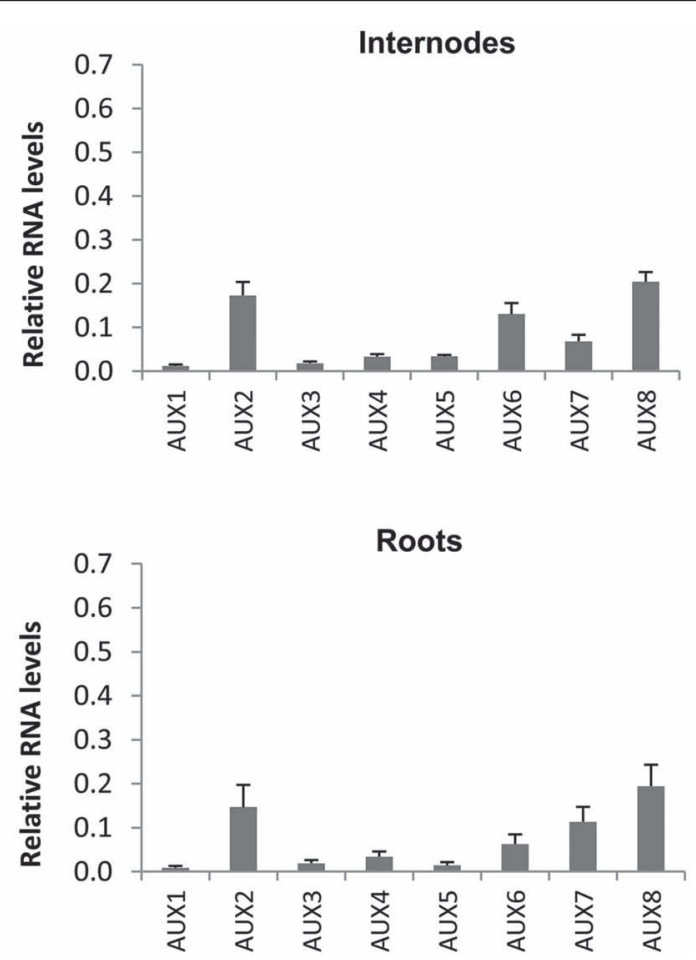

FIGURE 7 | Quantification of $\boldsymbol{A U X / L A X}$ transcripts expression by qRT-PCR. Most $A U X / L A X$ transcripts showed broad expression across plant tissues, including the previously undescribed PtaAUX4-8. PtaAUX2 and PtaAUX8 were highly expressed in internodes and developing xylem. Error bars represent the SEM.
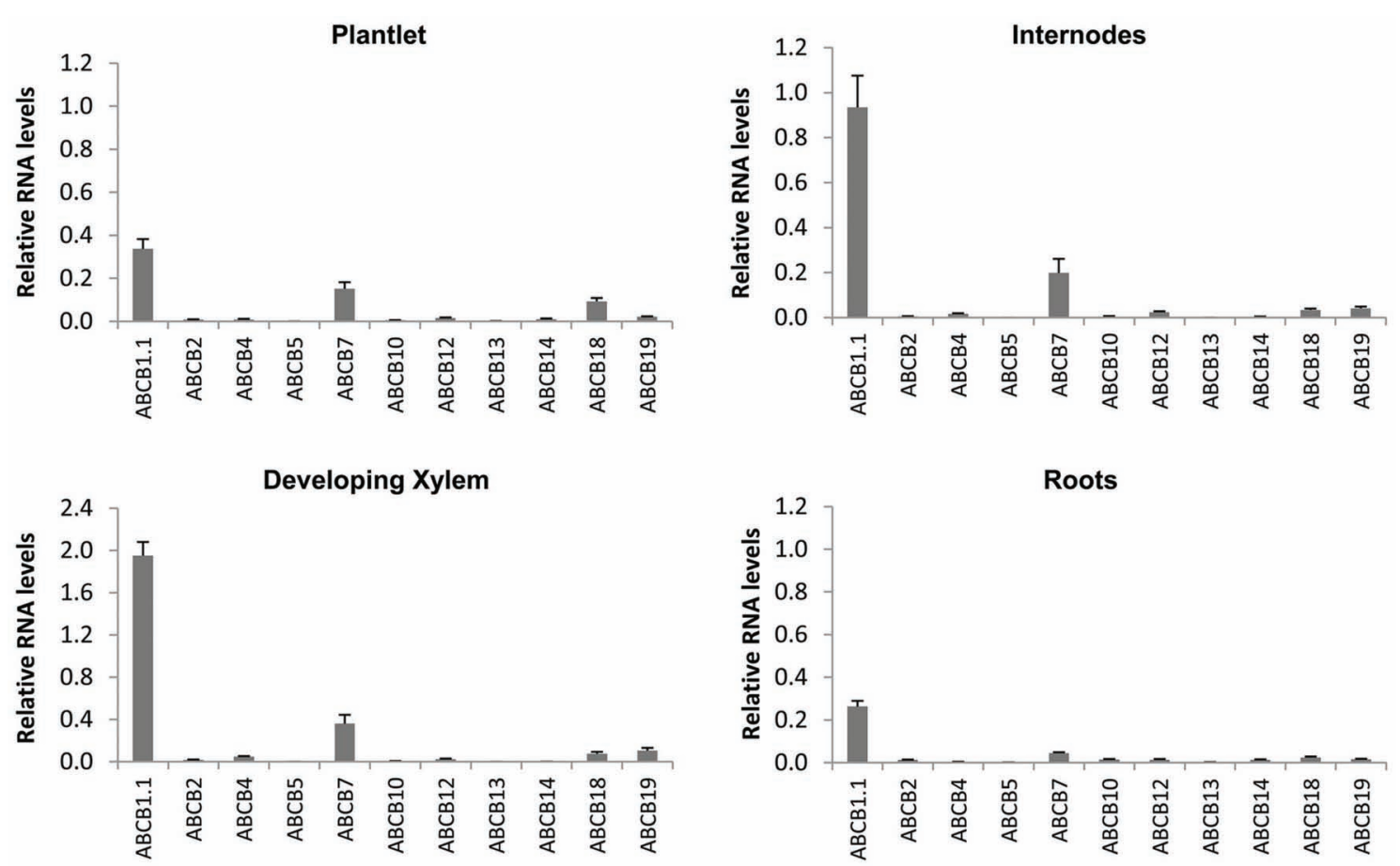

FIGURE 8 | Quantification of $A B C B$ transcripts expression by qRT-PCR. Most notable among the $A B C B$ family is PtaABCB1.1, which was highly expressed in internodes and developing xylem and whose ortholog in

Arabidopsis (AtABCB1) has been demonstrated to transport auxin. Expression patterns of all PtaABCB genes are previously undescribed. Error bars represent the SEM. 


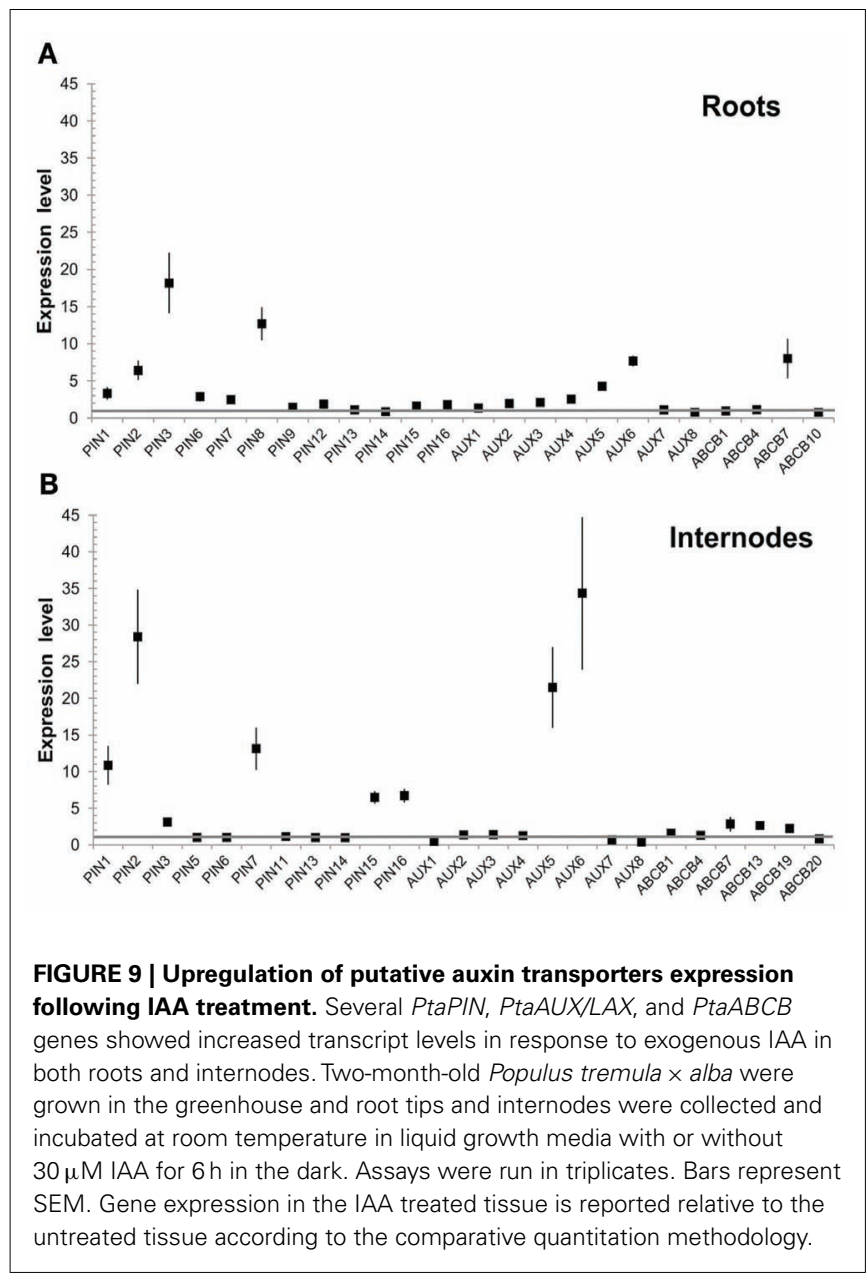

kingdoms (Jasinski et al., 2003; Verrier et al., 2008) and, as a group, are able to transport a wide array of different molecules (Geisler et al., 2005; Bandyopadhyay et al., 2007). Among the ABCs, the subclass $B$ includes proteins that are able to bind and transport auxin across the plasma membrane in Arabidopsis, whereas other members transport other substrates in addition to IAA (e.g., AtABCB14 functions primarily as a malate transporter (Lee et al., 2008)). There has been no functional characterization of the ABCBs in Populus to date and given the large size of the family and the likely role of one or more members in IAA transport, we sought to identify candidate PtrABCBs with this function. Our phylogenetic analysis shows that the coding sequences of $\operatorname{Ptr} A B C B 1.1$, PtrABCB1.2, and PtrABCB19 cluster together with $A t A B C B 1$ and AtABCB19 respectively, both of which are known IAA transporters with high specificity for IAA (Zazímalová et al., 2010). Interestingly, although 10 of the $20 \mathrm{PtaABCBs}$ are predicted to have one or more IAA binding sites based on tertiary structure, both PtrABCB1 and PtrABCB19 have only one clearly defined binding pocket for IAA. All but one of the remaining $A B C B s$ with putative IAA binding sites (PtrABCB2, PtrABCB5, PtrABCB6, PtrABCB8, PtrABCB11, PtrABCB14) cluster together in the same clade, which includes $A t A B C B 4$, a gene coding codes for another membrane protein capable of IAA transport (Terasaka et al., 2005; Kubeš et al., 2011). Similarly, PtrABCB16 occurs in the same clade as
AtABCB13 and AtABCB14, where AtABCB14 has been recently determined as responsible for auxin transport in the inflorescence stem of Arabidopsis (Kaneda et al., 2011).

We found PtrABCB1.1 to be highly expressed in most Populus tissues, particularly in internodes and developing xylem. PtrA$B C B 7$ was also expressed in these same tissues and was strongly upregulated in response to IAA, although most notably in roots. However, although coding sequence similarity places PtrABCB7 as a close relative of a presumed IAA transporter in Arabidopsis (AtABCB15; Kaneda et al., 2011), the protein was not predicted to contain an IAA binding site. We suggest therefore that PtrABCB1.1 and its nearly identical paralog PtrABCB1.2 are the most logical candidates for initial functional characterization, both in heterologous expression systems (e.g., Schizosaccharomyces pombe) and in planta, given their phylogenetic placement relative to AtABCB1 and predicted IAA binding sites. It is interesting to note that in contrast to AtABCB1 (Geisler et al., 2005), we did not find PtaABCB1.1 to be upregulated by exogenous IAA treatment. Lastly, we did not observe strong expression of PtaABCB19 in any Populus tissues nor was it upregulated by IAA. The expression of its presumed ortholog in Arabidopsis, AtABCB19, is induced by IAA treatments (Noh et al., 2001) and the protein often co-localizes with AtPIN1 (Bandyopadhyay et al., 2007), suggesting that the relationship of these two proteins may have changed. Clearly there is much to be learned about the role of these ABCBs in IAA transport in Populus.

\section{AUXIN TRANSPORTERS IN POPULUS STEM DEVELOPMENT}

That auxin regulates vascular development in woody plants is clear, but our understanding of the genetic mechanisms and the role of specific proteins in basipetal transport is limited. The expression of PttPIN1-3 and PttLAX1-3 has already been characterized in detail across the developing stem tissues of $P$. tremula $\times$ tremuloides (Schrader et al., 2003), but our results suggest that a far greater number of putative transporters are expressed in young internodes where cambial growth is being initiated. In particular, PtaPIN1, PtaPIN6, and PtaABCB1.1 are highly expressed in internodes, a complex tissue that includes primary xylem parenchyma, primary phloem, cortex, and a nascent vascular cambium. In developing xylem, PtaPIN1, PtaAUX2, and PtaABCB1.1 are highly expressed, with the latter likely to function in auxin transport given its protein sequence similarity to $A t A B C B 1$. Similarly, several previously uncharacterized transporters are strongly upregulated by auxin, including PtaPIN8, PtaAUX6, and PtaABCB7 in roots and PtaPIN7, PtaPIN15, PtaPIN16, PtaAUX5, and PtaAUX6 in internodes. Given the retention of copies of auxin transporters following duplication events, there is likely to be both redundancy and neo-functionalization for PAT proteins in Populus.

The vascular cambium and the secondary xylem and phloem that it produces are often viewed as distinct from primary growth, but it is important to remember that vascular development forms a continuum between stem and leaf (Spicer and Groover, 2010). We know a great deal about the role of PAT in venation patterning in leaves of Arabidopsis (Scarpella et al., 2006). Here, AtPIN1 directs auxin flow up through the epidermis toward a convergence point, from where it is channeled down through the center of a developing leaf primordium, establishing the location of the 
first central vascular bundle. This vascular bundle differentiates from a strand of procambium that is continuous with the vascular cambium below, such that the basipetal transport of auxin out of developing primordia is likely continuous with the basipetal stream moving down through the cambium (Lachaud and Bonnemain, 1984; Uggla et al., 1998; Kramer et al., 2008). Based on a combination of our results and published work in both Arabidopsis and Populus, we suggest that PtaPIN1, PtaAUX2, and PtaABCB1.1 are the best initial candidates for the maintenance of PAT in the cambial zone, although additional transporters are very likely involved. Given the slow time course and laborious nature of transformation in woody plants, our hope is that this work will provide a starting point for work in planta by identifying candidate IAA transporters involved in woody stem development. Functional studies, transport assays and protein localization are all needed to resolve the action of specific transporters in shaping the distribution of auxin across the cambial zone.

Finally, it is interesting to note that several members of the $P I N, A U X / L A X$, and $A B C B$ gene families are expressed in the mature xylem. Although the bulk of this tissue is dead (e.g., vessels and fibers), ray parenchyma cells remain alive for many years (Spicer and Holbrook, 2007) and serve as a route of transport between xylem and phloem (Van Bel, 1990). In particular, PtaPIN1, PtaAUX2, PtaAUX3, PtaAUX4 and PtaABCB1, PtaABCB7, PtaABCB20 were found to be expressed in these cells. In addition to their role in carbohydrate transport and storage, xylem parenchyma cells are able to exchange solutes with the transpiration stream and function in wound response. What is puzzling however is that these cells are symplasmically connected, at least in the radial direction, whereas PAT requires transport across a membrane. Furthermore, there is no evidence for free IAA in mature xylem (Uggla et al., 1996; Tuominen et al., 1997). Although conjugated forms of IAA are transported in the phloem (Baker, 2000) no studies to date have looked for conjugated IAA in ray or axial parenchyma in secondary xylem. Given their role in wound response, some capacity for IAA transport (or even IAA synthesis) would not be surprising, but transport assays and protein localization are needed to clarify any potential role these cells might play in IAA transport.

\section{THE ABCB GENE FAMILY DIVERSIFIED PRIOR TO THE PIN AND AUX/LAX FAMILIES AND PRIOR TO THE DIVERSIFICATION OF LAND PLANTS}

It is clear from our phylogenetic analysis that the $A B C B$ gene family existed before the diversification of land plants, whereas the PIN and $A U X / L A X$ families arose within the land plant clade. This is supported by the fact that $A B C B$ genes from a moss ( $P$. patens) and a lycopod ( $S$. moellendorffii) consistently occur nested within multiple, well-supported clades that also include higher plants (Figure 4; Figure A3 in Appendix). It also confirms previous work reconstructing the evolutionary history of this family (Bandyopadhyay et al., 2007; Krecek et al., 2009). In contrast, diversification of

\section{REFERENCES}

Bainbridge, K., Guyomarc'h, S., Bayer, E., Swarup, R., Bennett, M., Mandel, T., and Kuhlemeier, C. (2008). Auxin influx carriers stabilize phyllotactic patterning. Genes Dev. 22, $810-823$.

Baker, D. A. (2000). Long-distance vascular transport of endogenous hormones in plants and their role in

the PIN and AUX/LAX gene families occurred after the origin of land plants, as suggested by the well-supported and exclusively basal position of both Physcomitrella and Selaginella PIN and $A U X / L A X$ genes (Figures $\mathbf{2}$ and $\mathbf{3}$ ). There was already considerable diversity in the $A B C B$ gene family at the time of the monocot/dicot divergence, dated at approximately 130-150 Myr ago (Wolfe et al., 1989; Chaw et al., 2004; Bell et al., 2010), as we recovered as many as 10 distinct $A B C B$ gene clades that contain a clear monocot/dicot split with strong support. The picture is not as clear for the PIN and $A U X / L A X$ genes due to weak support at some nodes, but there may have been five copies of the PIN and likely just two copies of the $A U X / L A X$ genes at the time of the monocot/dicot divergence. It is not clear at this time whether all $A U X / L A X$ genes in monocots descended from a single original copy, as suggested by the tree found using aligned sequences under parsimony, since monocot $A U X / L A X$ genes were not recovered in a single clade in other trees (Figure 3).

In conclusion, we show that the deep history of the $\mathrm{ABCB}$ family of transporters coupled with the expansion of the PIN and AUX/LAX families following a genome duplication has led to a diverse array of over 40 putative auxin transport proteins in Populus. Given this large number and the inherent difficulties in working with a woody plant (e.g., long generation times, slow transformation process, difficult nucleic acid extraction), it is important to establish a comprehensive picture of gene expression profiles and predict their protein structures. By considering both evolutionary relationships and structural similarities to known auxin transporters, we can choose the most appropriate candidates for future study. One of the main goals in the short term should be to develop a set of tools for protein localization, including antibodies and protein fusions for stable plant transformation. Although technically difficult for trees, these findings should be coupled with functional studies with knockout mutants. Lastly, it will be important to determine the transport capacity and substrate specificity of target proteins of Populus by expressing them in heterologous systems such as $S$. pombe. We hope that this work provides a foundation on which to build an improved understanding of auxin transport in Populus, as knowing the role of specific transport proteins in secondary vascular development is likely key to enhanced utilization of woody plants.

\section{ACKNOWLEDGMENTS}

The authors would like to thank the laboratories of Noel M. Holbrook and Elena M. Kramer (Harvard University, OEB) for providing space and access to equipment, technical support, and for helpful discussion. The authors are also grateful to Angus S. Murphy and Wendy A. Peer (Purdue University) for helpful discussion of the manuscript; Serena Varotto and Cristian Forestan for sharing sequences and for helpful discussion. This work was supported by a Rowland Junior Fellowship awarded to Rachel Spicer from 2007 to 2010 .

source: sink regulation. Isr. J. Plant Sci. 48, 199-203.

Bandyopadhyay, A., Blakeslee, J. J., Lee, O. R., Mravec, J., Sauer, M., Titapiwatanakun, B., Makam, S. N.,
Bouchard, R., Geisler, M., Martinoia, E., Friml, J., Peer, W. A., and Murphy, A. S. (2007). Interactions of PIN and PGP auxin transport mechanisms. Biochem. Soc. Trans. 35, 137-141. 
Banks, J. A., Nishiyama, T., Hasebe, M., Bowman, J. L., Gribskov, M., DePamphilis, C., Albert, V. A., Aono, N., Aoyama, T., Ambrose, B. A., Ashton, N. W., Axtell, M. J., Barker, E., Barker, M. S., Bennetzen, J. L., Bonawitz, N. D., Chapple, C., Cheng, C., Correa, L. G., Dacre, M., DeBarry, J., Dreyer, I., Elias, M., Engstrom, E. M., Estelle, M., Feng, L., Finet, C., Floyd, S. K., Frommer, W. B., Fujita, T., Gramzow, L., Gutensohn, M., Harholt, J., Hattori, M., Heyl, A., Hirai, T., Hiwatashi, Y., Ishikawa, M., Iwata, M., Karol, K. G., Koehler, B., Kolukisaoglu, U., Kubo, M., Kurata, T., Lalonde, S., Li, K., Li, Y., Litt, A., Lyons, E., Manning, G., Maruyama, T., Michael, T. P., Mikami, K., Miyazaki, S., Morinaga, S., Murata, T., Mueller-Roeber, B., Nelson, D. R., Obara, M., Oguri, Y., Olmstead, R. G., Onodera, N., Petersen, B. L., Pils, B., Prigge, M., Rensing, S. A., Riaño-Pachón, D. M., Roberts, A. W., Sato, Y., Scheller, H. V., Schulz, B., Schulz, C., Shakirov, E. V., Shibagaki, N., Shinohara, N., Shippen, D. E., Sørensen, I., Sotooka, R., Sugimoto, N., Sugita, M., Sumikawa, N., Tanurdzic, M., Theissen, G., Ulvskov, P., Wakazuki, S., Weng, J. K., Willats, W. W., Wipf, D., Wolf, P. G., Yang, L., Zimmer, A. D., Zhu, Q., Mitros, T., Hellsten, U., Loqué, D., Otillar, R., Salamov, A., Schmutz, J., Shapiro, H., Lindquist, E., Lucas, S., Rokhsar, D., and Grigoriev, I. V. (2011). The Selaginella genome identifies genetic changes associated with the evolution of vascular plants. Science 332, 960-963.

Bell, C. D., Soltis, D. E., and Soltis, P. S. (2010). The age and diversification of the angiosperms re-revisited. Am. J. Bot. 97, 1296-1303.

Benková, E., Michniewicz, M., Sauer, M., Teichmann, T., Seifertová, D., Jürgens, G., and Friml, J. (2003). Local, efflux-dependent auxin gradients as a common module for plant organ formation. Cell 115, 591-602.

Bennett, M. J., Marchant, A., Green, H. G., May, S. T., Ward, S. P., Millner, P. A., Walker, A. R., Schulz, B., and Feldmann, K. A. (1996). Arabidopsis AUX1 gene: a permease-like regulator of root gravitropism. Science 273, 948-950.

Björklund, S., Antti, H., Uddestrand, I., Moritz, T., and Sundberg, B. (2007). Cross-talk between gibberellin and auxin in development of Populus wood: gibberellin stimulates polar auxin transport and has a common transcriptome with auxin. Plant $J$. 52, 499-511.
Brunner, A. M., Yakovlev, I. A., and Strauss, S. H. (2004). Validating internal controls for quantitative plant gene expression studies. BMC Plant Biol. 4, 14. doi:10.1186/1471-2229-4-14

Carraro, N., Forestan, C., Canova, S., Traas, J., and Varotto, S. (2006). ZmPINla and ZmPIN1b encode two novel putative candidates for polar auxin transport and plant architecture determination of maize. Plant Physiol. 142, 254-264.

Castresana, J. (2000). Selection of conserved blocks from multiple alignments for their use in phylogenetic analysis. Mol. Biol. Evol. 17, 540-552.

Chaw, S.-M., Chang, C.-C., Chen, H.L., and Li, W.-H. (2004). Dating the monocot-dicot divergence and the origin of core eudicots using whole chloroplast genomes. J. Mol. Evol.58, 424-441.

Chen, R., Hilson, P., Sedbrook, J., Rosen, E., Caspar, T., and Masson, P. H. (1998). The Arabidopsis thaliana AGRAVITROPIC 1 gene encodes a component of the polar-auxintransport efflux carrier. Proc. Natl. Acad. Sci. U.S.A. 95, 15112-15117.

Dolinsky, T. J., Czodrowski, P., Li, H., Nielsen, J. E., Jensen, J. H., Klebe, G., and Baker, N. A. (2007). PDB2PQR: expanding and upgrading automated preparation of biomolecular structures for molecular simulations. Nucleic Acids Res. 35, W522-W525.

Dolinsky, T. J., Nielsen, J. E., McCammon, J. A., and Baker, N. A. (2004). PDB2PQR: an automated pipeline for the setup of Poisson-Boltzmann electrostatics calculations. Nucleic Acids Res. 32, W665-W667.

Forestan, C., Meda, S., and Varotto, S. (2010). ZmPIN1-mediated auxin transport is related to cellular differentiation during maize embryogenesis and endosperm development. Plant Physiol. 152, 1373-1390.

Friml, J., Benková, E., Blilou, I., Wisniewska, J., Hamann, T., Ljung, K., Woody, S., Sandberg, G., Scheres, B., Jürgens, G., and Palme, K. (2002a). AtPIN4 mediates sink-driven auxin gradients and root patterning in Arabidopsis. Cell 108, 661-673.

Friml, J., Wisniewska, J., Benková, E., Mendgen, K., and Palme, K. (2002b). Lateral relocation of auxin efflux regulator PIN3 mediates tropism in Arabidopsis. Nature 415, 806-809.

Friml, J., and Jones, A. R. (2010). Endoplasmic reticulum: the rising compartment in auxin biology. Plant Physiol. 154, 458-462.

Friml, J., Vieten, A., Sauer, M., Weijers, D., Schwarz, H., Hamann, T.,
Offringa, R., and Jürgens, G. (2003). Efflux-dependent auxin gradients establish the apical-basal axis of Arabidopsis. Nature 426, 147-153.

Gälweiler, L., Guan, C., Müller, A., Wisman, E., Mendgen, K., Yephremov, A., and Palme, K. (1998). Regulation of polar auxin transport by AtPIN1 in Arabidopsis vascular tissue. Science 282, 2226-2230.

Geisler, M., Blakeslee, J. J., Bouchard, R., Lee, O. R., Vincenzetti, V., Bandyopadhyay, A., Titapiwatanakun, B. Peer, W. A., Bailly, A., Richards, E. L., Ejendal, K. F., Smith, A. P., Baroux C., Grossniklaus, U., Müller, A., Hrycyna, C. A., Dudler, R., Murphy, A. S., and Martinoia, E.(2005). Cellular efflux of auxin catalyzed by the Arabidopsis MDR/PGP transporter AtPGP1. Plant J. 44, 179-194.

Guo, A.-Y., Zhu, Q.-H., Chen, X., and Luo, J.-C. (2007). GSDS: a gene structure display server. Yi Chuan 29, 1023-1026.

Gutierrez, L., Mauriat, M., Guénin, S., Pelloux, J., Lefebvre, J.-F., Louvet, R., Rusterucci, C., Moritz, T. Guerineau, F., Bellini, C., and Van Wuytswinkel, O. (2008). The lack of a systematic validation of reference genes: a serious pitfall undervalued in reverse transcription-polymerase chain reaction (RT-PCR) analysis in plants. Plant Biotechnol. J. 6, 609-618.

Hellgren, J. M., Olofsson, K., Plant, U., Centre, S., and Sciences, A. (2004). Patterns of auxin distribution during gravitational induction of reaction wood in poplar and pine. Plant Physiol. 135, 212-220.

Jasinski, M., Ducos, E., Martinoia, E., and Boutry, M. (2003). The ATPbinding cassette transporters: structure, function, and gene family comparison between. Plant Physiol. 131, 1169-1177.

Kaneda, M., Schuetz, M., Lin, B. S. P., Chanis, C., Hamberger, B., Western, T. L., Ehlting, J., and Samuels, A, L. (2011). ABC transporters coordinately expressed during lignification of Arabidopsis stems include a set of ABCBs associated with auxin transport. J. Exp. Bot. 62, 2063-2077.

Katekar, G. F., and Geissler, A. E. (1980). Auxin transport inhibitors. Plant Physiol. 66, 1190-1195.

Katoh, K., Asimenos, G., and Toh, H. (2009). Multiple alignment of DNA sequences with MAFFT. Methods Mol. Biol. 537, 39-64.

Knöller, A. S., Blakeslee, J. J., Richards, E. L., Peer, W. A., and Murphy, A. S. (2010). Brachytic2/ZmABCB1 functions in IAA export from intercalary meristems. J. Exp. Bot. 61 3689-3696.

Kramer, E. M., Dorit, R. L., and Irish, V. F. (1998). Molecular evolution of genes controlling petal and stamen development: duplication and divergence within the APETALA3 and PISTILLATA MADS-box gene lineages. Genetics 149, 765-783.

Kramer, E. M., Lewandowski, M., Beri, S., Bernard, J., Borkowski, M., Borkowski, M. H., Burchfield, L. A., Mathisen, B., and Normanly, J. (2008). Auxin gradients are associated with polarity changes in trees. Science 320, 1610

Kubeš, M., Yang, H., Richter, G. L., Cheng, Y., Mlodzinska, E., Wang, X., Blakeslee, J. J., Carraro, N., Petrášek, J., Zažímalová, E., Hoyerová, K., Peer, W. A., and Murphy, A. S. (2011). The Arabidopsis concentrationdependent influx/efflux transporter ABCB4 regulates cellular auxin levels in the root epidermis. Plant J. [Epub ahead of print].

Krecek, P., Skupa, P., Libus, J., Naramoto, S., Tejos, R., Friml, J., and Zažímalová, E. (2009). Protein family review The PIN-FORMED (PIN) protein family of auxin transporters. Genome Biol. 10, 1-11.

Lachaud, S., and Bonnemain, J. L. (1984). Seasonal variations in the polar transport pathways and retention sites of $\left[{ }^{3} \mathrm{H}\right]$ indole-3-acetic acid in young branches of Fagus sylvatica L. Planta 161, 207-215.

Lee, B. H. A., Johnston, R., Yang, Y., Gallavotti, A., Kojima, M., Travençolo, B. A. N., Costa, L. D. F., Sakakibara, H., and Jackson, D. (2009). Studies of aberrant phyllotaxy1 mutants of maize indicate complex interactions between auxin and cytokinin signaling in the shoot apical meristem. Plant Physiol. 150, 205-216.

Lee, M., Choi, Y., Burla, B., Kim, Y.-Y., Jeon, B., Maeshima, M., Yoo, J.-Y., Martinoia, E., and Lee, Y. (2008). The $\mathrm{ABC}$ transporter AtABCB14 is a malate importer and modulates stomatal response to CO2. Nat. Cell Biol. 10, 1217-1223.

Livak, K. J., and Schmittgen, T. D. (2001). Analysis of relative gene expression data using real-time quantitative PCR and the 2(-Delta Delta $\mathrm{C}(\mathrm{T})$ ) method. Methods 25, 402-408.

Ljung, K., Bhalerao, R. P., and Sandberg, G. (2001a). Sites and homeostatic control of auxin biosynthesis in Arabidopsis during vegetative growth. Plant J. 28, 465-474. 
Ljung, K., Ostin, A., Lioussanne, L., and Sandberg, G. (2001b). Developmental regulation of indole-3-acetic acid turnover in Scots pine seedlings. Plant Physiol. 125, 464-475.

Ljung, K., Hull, A. K., Celenza, J., Yamada, M., Estelle, M., and Normanly, J. (2005). Sites and regulation of auxin biosynthesis in Arabidopsis roots. Plant Cell 17, 1090-1104.

Lomax, T., Muday, G. K., and Rubery, P. H. (1995). Plant Hormones: Physiology, Biochemistry, and Molecular Biology. Dordrecht: K. A. Publishers.

Luschnig, C., Gaxiola, R. A., Grisafi, P., and Fink, G. R. (1998). EIR1, a rootspecific protein involved in auxin transport, is required for gravitropism in Arabidopsis thaliana. Genes Dev. 12, 2175-2187.

Merchant, S. S., Prochnik, S. E., Vallon, O., Harris, E. H., Karpowicz, S. J., Witman, G. B., Terry, A., Salamov, A., Fritz-Laylin, L. K., Maréchal-Drouard, L., Marshall, W. F., Qu, L. H., Nelson, D. R., Sanderfoot, A. A., Spalding, M. H., Kapitonov, V. V., Ren, Q., Ferris, P., Lindquist, E., Shapiro, H., Lucas, S. M., Grimwood, J., Schmutz, J., Cardol, P., Cerutti, H., Chanfreau, G., Chen, C. L., Cognat, V., Croft, M. T., Dent, R., Dutcher, S., Fernández, E., Fukuzawa, H., GonzálezBallester, D., González-Halphen, D., Hallmann, A., Hanikenne, M., Hippler, M., Inwood, W., Jabbari, K., Kalanon, M., Kuras, R., Lefebvre, P. A., Lemaire, S. D., Lobanov, A. V., Lohr, M., Manuell, A., Meier, I., Mets, L., Mittag, M., Mittelmeier, T., Moroney, J. V., Moseley, J., Napoli, C., Nedelcu, A. M., Niyogi, K., Novoselov, S. V., Paulsen, I. T., Pazour, G., Purton, S., Ral, J. P., Riaño-Pachón, D. M., Riekhof, W., Rymarquis, L., Schroda, M., Stern, D., Umen, J., Willows, R., Wilson, N., Zimmer, S. L., Allmer, J., Balk, J., Bisova, K., Chen, C. J., Elias, M., Gendler, K., Hauser, C., Lamb, M. R., Ledford, H., Long, J. C., Minagawa, J., Page, M. D., Pan, J., Pootakham, W., Roje, S., Rose, A., Stahlberg, E., Terauchi, A. M., Yang, P., Ball, S., Bowler, C., Dieckmann, C. L., Gladyshev, V. N., Green, P., Jorgensen, R., Mayfield, S., Mueller-Roeber, B., Rajamani, S., Sayre, R. T., Brokstein, P., Dubchak, I., Goodstein, D., Hornick, L., Huang, Y. W., Jhaveri, J., Luo, Y., Martínez, D., Ngau, W. C., Otillar, B., Poliakov, A., Porter, A., Szajkowski, L., Werner, G., Zhou, K., Grigoriev, I. V., Rokhsar, D. S., and Grossman, A. R. (2007). The Chlamydomonas genome reveals the evolution of key animal and plant functions. Science 318, 245-250.

Miller, M. A., Pfeiffer, W., and Schwartz, T. (2010). "Creating the CIPRES science gateway for inference of large phylogenetic trees," in Gateway Computing Environments Workshop (GCE), New Orleans.

Mravec, J., Skupa, P., Bailly, A., Hoyerová, K., Krecek, P., Bielach, A., Petrásek, J., Zhang, J., Gaykova, V., Stierhof, Y.-D., Dobrev, P. I., Schwarzerová, K., Rolcík, J., Seifertová, D., Luschnig, C., Benková, E., Zazímalová, E., Geisler, M., and Friml, J. (2009). Subcellular homeostasis of phytohormone auxin is mediated by the ER-localized PIN5 transporter. Nature 459, 1136-1140.

Müller, A., Guan, C., Gälweiler, L., Tänzler, P., Huijser, P., Marchant, A., Parry, G., Bennett, M., Wisman, E., and Palme, K. (1998). AtPIN2 defines a locus of Arabidopsis for root gravitropism control. EMBO J. 17, 6903-6911.

Multani, D. S., Briggs, S. P., Chamberlin, M. A, Blakeslee, J. J., Murphy, A. S., and Johal, G. S. (2003). Loss of an MDR transporter in compact stalks of maize br2 and sorghum dw3 mutants. Science 302, 81-84.

Nilsson, J., Karlberg, A., Antti, H., Lopez-Vernaza, M., Mellerowicz, E., Perrot-Rechenmann, C., Sandberg, G., and Bhalerao, R. P. (2008). Dissecting the molecular basis of the regulation of wood formation by auxin in hybrid aspen. Plant Cell 20, 843-855.

Noh, B., Murphy, A. S., and Spalding, E. P. (2001). Multidrug resistancelike genes of Arabidopsis required for auxin transport and auxinmediated development. Plant Cell 13, 2441-2454.

Okada, K., Ueda, J., Komaki, M. K., Bell, C. J., and Shimura, Y. (1991). Requirement of the auxin polar transport system in early stages of Arabidopsis floral bud formation. Plant Cell 3, 677.

Petrásek, J., and Friml, J. (2009). Auxin transport routes in plant development. Development 136, 2675-2688.

Pfaffl, M. W. (2001). A new mathematical model for relative quantification in real-time RT-PCR. Nucleic Acids Res. 29, e45.

Prochnik, S. E., Umen, J., Nedelcu, A. M., Hallmann, A., Miller, S. M., Nishii, I., Ferris, P., Kuo, A., Mitros, T., Fritz-Laylin, L. K., Hellsten, U., Chapman, J., Simakov, O., Rensing, S. A., Terry, A., Pangilinan, J., Kapitonov, V., Jurka, J., Salamov, A., Shapiro, H., Schmutz,
J., Grimwood, J., Lindquist, E., Lucas, S., Grigoriev, I. V., Schmitt, R., Kirk, D., and Rokhsar, D. S. (2010). Genomic analysis of organismal complexity in the multicellular green alga Volvox carteri. Science 329, 223-226.

Rea, P. A. (2007). Plant ATP-binding cassette transporters. Annu. Rev. Plant Biol. 58, 347-375.

Rensing, S. A., Lang, D., Zimmer, A. D., Terry, A., Salamov, A., Shapiro, H., Nishiyama, T., Perroud, P.-F. Lindquist, E. A., Kamisugi, Y., Tanahashi, T., Sakakibara, K., Fujita, T. Oishi, K., Shin-I, T., Kuroki, Y., Toyoda, A., Suzuki, Y., Hashimoto, S. Yamaguchi, K., Sugano, S., Kohara, Y., Fujiyama, A., Anterola, A., Aoki, S., Ashton, N., Barbazuk, W. B., Barker, E., Bennetzen, J. L., Blankenship, R., Cho, S. H., Dutcher, S. K., Estelle, M., Fawcett, J. A., Gundlach, H., Hanada, K., Heyl, A., Hicks, K. A., Hughes, J., Lohr, M., Mayer, K. Melkozernov, A., Murata, T., Nelson, D. R., Pils, B., Prigge, M., Reiss, B., Renner, T., Rombauts, S., Rushton, P. J., Sanderfoot, A., Schween, G., Shiu, S. H., Stueber, K., Theodoulou, F. L., Tu, H., Van de Peer, Y., Verrier, P. J. Waters, E., Wood, A., Yang, L., Cove, D., Cuming, A. C., Hasebe, M., Lucas, S., Mishler, B. D., Reski, R., Grigoriev, I. V., Quatrano, R. S., and Boore, J. L. (2008). The Physcomitrella genome reveals evolutionary insights into the conquest of land by plants. Science 319, 64-69.

Sanchez-Fernandez, R., Davies, T. G., Coleman, J. O., and Rea, P. A. (2001). The Arabidopsis thaliana ABC protein superfamily, a complete inventory. J. Biol. Chem. 276, 30231-30244.

Sauer, M., Balla, J., Luschnig, C., Wisniewska, J., Reinöhl, V., Friml, J., and Benková, E. (2006). Canalization of auxin flow by Aux/IAAARF-dependent feedback regulation of PIN polarity. Genes Dev. 20, 2902-2911.

Scarpella, E., Marcos, D., Friml, J., and Berleth, T. (2006). Control of leaf vascular patterning by polar auxin transport. Genes Dev. 20, 1015-1027.

Schrader, J., Baba, K., May, S. T., Palme, K., Bennett, M., Bhalerao, R. P., and Sandberg, G. (2003). Polar auxin transport in the wood-forming tissues of hybrid aspen is under simultaneous control of developmental and environmental signals. Proc. Natl. Acad. Sci. U.S.A. 100, 10096-10101.

Schrader, J., Moyle, R., Bhalerao, R., Hertzberg, M., Lundeberg, J.,
Nilsson, P., and Bhalerao, R. P. (2004). Cambial meristem dormancy in trees involves extensive remodelling of the transcriptome. Plant J. 40, 173-187.

Secchi, F., MacIver, B., Zeidel, M. L., and Zwieniecki, M. A. (2009). Functional analysis of putative genes encoding the PIP2 water channel subfamily in Populus trichocarpa. Tree Physiol. 29, 1467-1477.

Shen, C., Bai, Y., Wang, S., Zhang, S., Wu, Y., Chen, M., Jiang, D., and Qi, Y. (2010). Expression profile of PIN, AUX/LAX and PGP auxin transporter gene families in Sorghum bicolor under phytohormone and abiotic stress. FEBS J. 277, 2954-2969.

Sidler, M., Hassa, P., Hasan, S., Ringli, C., and Dudler, R. (1998). Involvement of an $\mathrm{ABC}$ transporter in a developmental pathway regulating hypocotyl cell elongation in the light. Plant Cell 10, 1623-1636.

Spicer, R., and Groover, A. (2010). Evolution of development of vascular cambia and secondary growth. New Phytol. 186, 577-592.

Spicer, R., and Holbrook, N. M. (2007). Parenchyma cell respiration and survival in secondary xylem: does metabolic activity decline with cell age? Plant Cell Environ. 30, 934-943.

Stamatakis, A., Hoover, P., and Rougemont, J. (2008). A rapid bootstrap algorithm for the RAxML Web servers. Syst. Biol. 57, 758-771.

Sussman, M. R., and Goldsmith, M. H. M. (1981). The action of specific inhibitors of auxin transport on uptake of auxin and binding of $\mathrm{N}$-1-naphthylphthalamic acid to a membrane site in maize coleoptiles. Planta 13-18.

Swarup, K., Benková, E., Swarup, R., Casimiro, I., Péret, B., Yang, Y., Parry, G., Nielsen, E., De Smet, I., Vanneste, S., Levesque, M. P., Carrier, D., James, N., Calvo, V., Ljung, K., Kramer, E., Roberts, R., Graham, N., Marillonnet, S., Patel, K., Jones, J. D., Taylor, C. G., Schachtman, D. P., May, S., Sandberg, G., Benfey, P., Friml, J., Kerr, I., Beeckman, T., Laplaze, L., and Bennett, M. J. (2008). The auxin influx carrier LAX3 promotes lateral root emergence. Nat. Cell Biol. 10, 946-954.

Swarup, R., Friml, J., Marchant, A., Ljung, K., Sandberg, G., Palme, K., and Bennett, M. (2001). Localization of the auxin permease AUX1 suggests two functionally distinct hormone transport pathways operate in the Arabidopsis root apex. Genes Dev. 15, 2648-2653. 
Swarup, R., Kramer, E. M., Perry, P., Knox, K., Leyser, H. M. O., Haseloff, J., Beemster, G. T. S., Bhalerao, R., and Bennett, M. J. (2005). Root gravitropism requires lateral root cap and epidermal cells for transport and response to a mobile auxin signal. Nat. Cell Biol. 7, 1057-1065.

Talavera, G., and Castresana, J. (2007). Improvement of phylogenies after removing divergent and ambiguously aligned blocks from protein sequence alignments. Syst. Biol. 56, 564-577.

Terasaka, K., Blakeslee, J. J., Titapiwatanakun, B., Peer, W. A., Bandyopadhyay, A., Makam, S. N., Lee, R., Richards, E. L., Murphy, A. S., Sato, F., and Yazaki, K. (2005). PGP4, an ATP binding cassette P-glycoprotein, catalyzes auxin transport in Arabidopsis thaliana roots. Plant Cell 17, 2922-2939.

Titapiwatanakun, B., and Murphy, A. S. (2009). Post-transcriptional regulation of auxin transport proteins: cellular trafficking, protein phosphorylation, protein maturation, ubiquitination, and membrane composition. J. Exp. Bot. 60, 1093-1107.

Tuominen, H., Puech, L., Fink, S., and Sundberg, B. (1997). A radial concentration gradient of indole-3acetic acid is related to secondary xylem development in hybrid aspen. Plant Physiol. 115, 577-585.

Tuskan, G. A., Difazio, S., Jansson, S., Bohlmann, J., Grigoriev, I., Hellsten, U., Putnam, N., Ralph, S., Rombauts, S., Salamov, A., Schein, J., Sterck, L., Aerts, A., Bhalerao, R. R., Bhalerao, R. P., Blaudez, D., Boerjan, W., Brun, A., Brunner, A., Busov, V., Campbell, M., Carlson, J., Chalot, M., Chapman, J., Chen, G. L., Cooper, D., Coutinho, P. M., Couturier, J., Covert, S., Cronk, Q., Cunningham, R., Davis, J., Degroeve, S., Déjardin, A., Depamphilis, C., Detter, J., Dirks, B., Dubchak, I., Duplessis, S., Ehlting, J., Ellis, B., Gendler, K., Goodstein, D., Gribskov, M., Grimwood, J., Groover, A., Gunter, L., Hamberger, B., Heinze, B., Helariutta, Y., Henrissat, B., Holligan, D., Holt, R., Huang, W., Islam-Faridi, N., Jones, S., Jones-Rhoades, M., Jorgensen, R., Joshi, C., Kangasjärvi, J., Karlsson,
J., Kelleher, C., Kirkpatrick, R., Kirst, M., Kohler, A., Kalluri, U., Larimer, F., Leebens-Mack, J., Leplé, J. C., Locascio, P., Lou, Y., Lucas, S., Martin, F., Montanini, B., Napoli, C., Nelson, D. R., Nelson, C., Nieminen, K., Nilsson, O., Pereda, V., Peter, G., Philippe, R., Pilate, G., Poliakov, A., Razumovskaya, J., Richardson, P., Rinaldi, C., Ritland, K., Rouzé, P., Ryaboy, D., Schmutz, J., Schrader, J., Segerman, B., Shin, H., Siddiqui, A., Sterky, F., Terry, A., Tsai, C. J., Uberbacher, E., Unneberg, P., Vahala, J., Wall, K., Wessler, S., Yang, G., Yin, T., Douglas, C., Marra, M., Sandberg, G., Van de Peer, Y., and Rokhsar, D. (2006). The genome of black cottonwood, Populus trichocarpa (Torr. \& Gray). Science 313, 1596-1604.

Uggla, C., Mellerowicz, E., and Sundberg, B. (1998). Indole-3-acetic acid controls cambial growth in scots pine by positional signaling. Plant Physiol. 117, 113-121.

Uggla, C., Moritz, T., Sandberg, G., and Sundberg, B. (1996). Auxin as a positional signal in pattern formation in plants. Proc. Natl. Acad. Sci. U.S.A. 93, 9282-9286.

Utsuno, K., Shikanai, T., Yamada, Y., and Hashimoto, T. (1998). Agr, an Agravitropic locus of Arabidopsis thaliana, encodes a novel membrane-protein family member. Plant Cell Physiol. 39, 1111-1118.

Van Bel, A. J. E. (1990). Xylem-phloem exchange via the rays: the undervalued route of transport. J. Exp. Bot. 41, 631-644.

Vandesompele, J., De Preter, K., Pattyn, F., Poppe, B., Van Roy, N., De Paepe, A., and Speleman, F. (2002). Accurate normalization of real-time quantitative RT-PCR data by geometric averaging of multiple internal control genes. Genome Biol. 3, RESEARCH0034.

Varón, A., Vinh, L. S., and Wheeler, W. C. (2009). POY version 4: phylogenetic analysis using dynamic homologies. Cladistics 26, 72-85.

Vernoux, T., Brunoud, G., Farcot, E., Morin, V., Van den Daele, H., Legrand, J., Oliva, M., Das, P., Larrieu, A., Wells, D., Guédon, Y., Armitage, L., Picard, F.,
Guyomarc'h, S., Cellier, C., Parry, G., Koumproglou, R., Doonan, J. H., Estelle, M., Godin, C., Kepinski, S., Bennett, M., De Veylder, L., and Traas, J. (2011). The auxin signalling network translates dynamic input into robust patterning at the shoot apex. Mol. Syst. Biol. 7, 508 .

Verrier, P. J., Bird, D., Burla, B., Dassa, E., Forestier, C., Geisler, M., Klein, M., Kolukisaoglu, U., Lee, Y., Martinoia, E., Murphy, A., Rea, P. A., Samuels, L., Schulz, B., Spalding, E. J., Yazaki, K., and Theodoulou, F. L. (2008). Plant ABC proteins - a unified nomenclature and updated inventory. Trends Plant Sci. 13, 151-159.

Vieten, A., Vanneste, S., Wisniewska, J., Benková, E., Benjamins, R., Beeckman, T., Luschnig, C., and Friml, J. (2005). Functional redundancy of PIN proteins is accompanied by auxin-dependent crossregulation of PIN expression. Development 132, 4521-4531.

Wang, J.-R., Hu, H., Wang, G.-H., Li, J., Chen, J.-Y., and Wu, P. (2009). Expression of PIN genes in rice (Oryza sativa L.): tissue specificity and regulation by hormones. $\mathrm{Mol}$. Plant 2, 823-831.

Wheeler, W. (1996). Optimization alignment: the end of multiple sequence alignment in phylogenetics? Cladistics 12, 1-9.

Wiederstein, M., and Sippl, M. J. (2007). ProSA-web: interactive web service for the recognition of errors in three-dimensional structures of proteins. Nucleic Acids Res. 35, W407-W410

Wolfe, K. H., Gouy, M., Yang, Y. W. Sharp, P. M., and Li, W. H. (1989). Date of the monocot-dicot divergence estimated from chloroplast DNA sequence data. Proc. Natl. Acad. Sci. U.S.A. 86, 6201-6205.

Wu, G., Lewis, D. R., and Spalding, E. P. (2007). Mutations in Arabidopsis multidrug resistance-like $\mathrm{ABC}$ transporters separate the roles of acropetal and basipetal auxin transport in lateral root development. Plant Cell 19, 1826-1837.

$\mathrm{Wu}, \mathrm{X}$., and McSteen, P. (2007). The role of auxin transport during inflorescence development in maize (Zea mays, Poaceae). Am. J. Bot. 94, 1745-1755.

Yang, H., and Murphy, A. S. (2009). Functional expression and characterization of Arabidopsis ABCB, AUX 1 and PIN auxin transporters in Schizosaccharomyces pombe. Plant J. 59, 179-191.

Yang, Y., Hammes, U. Z., Taylor, C. G., Schachtman, D. P., and Nielsen, E. (2006). High-affinity auxin transport by the AUX1 influx carrier protein. Curr. Biol. 16, 1123-1127.

Yemm, A., May, S., Williams, L., Millner, P., Tsurumi, S., Moore, I., Napier, R., Kerr, I. D., and Bennett, M. J. (2004). Structure-function analysis of the presumptive Arabidopsis auxin permease AUX1. Plant Cell 16, 3069-3083.

Zazímalová, E., Murphy, A. S., Yang, H., Hoyerová, K., and Hosek, P. (2010). Auxin transporters - why so many? Cold Spring Harb. Perspect. Biol. 2, $1-14$.

Conflict of Interest Statement: The authors declare that the research was conducted in the absence of any commercial or financial relationships that could be construed as a potential conflict of interest.

Received: 29 October 2011; accepted: 17 January 2012; published online: $07 \mathrm{Feb}$ ruary 2012.

Citation: Carraro N, Tisdale-Orr TE Clouse RM, Knöller AS and Spicer $R$ (2012) Diversification and expression of the PIN, AUX/LAX, and ABCB families of putative auxin transporters in Populus. Front. Plant Sci. 3:17. doi: 10.3389/fpls.2012.00017

This article was submitted to Frontiers in Plant Physiology, a specialty of Frontiers in Plant Science.

Copyright () 2012 Carraro, Tisdale-Orr, Clouse, Knöller and Spicer. This is an open-access article distributed under the terms of the Creative Commons Attribution Non Commercial License, which permits non-commercial use, distribution, and reproduction in other forums, provided the original authors and source are credited. 


\section{APPENDIX}

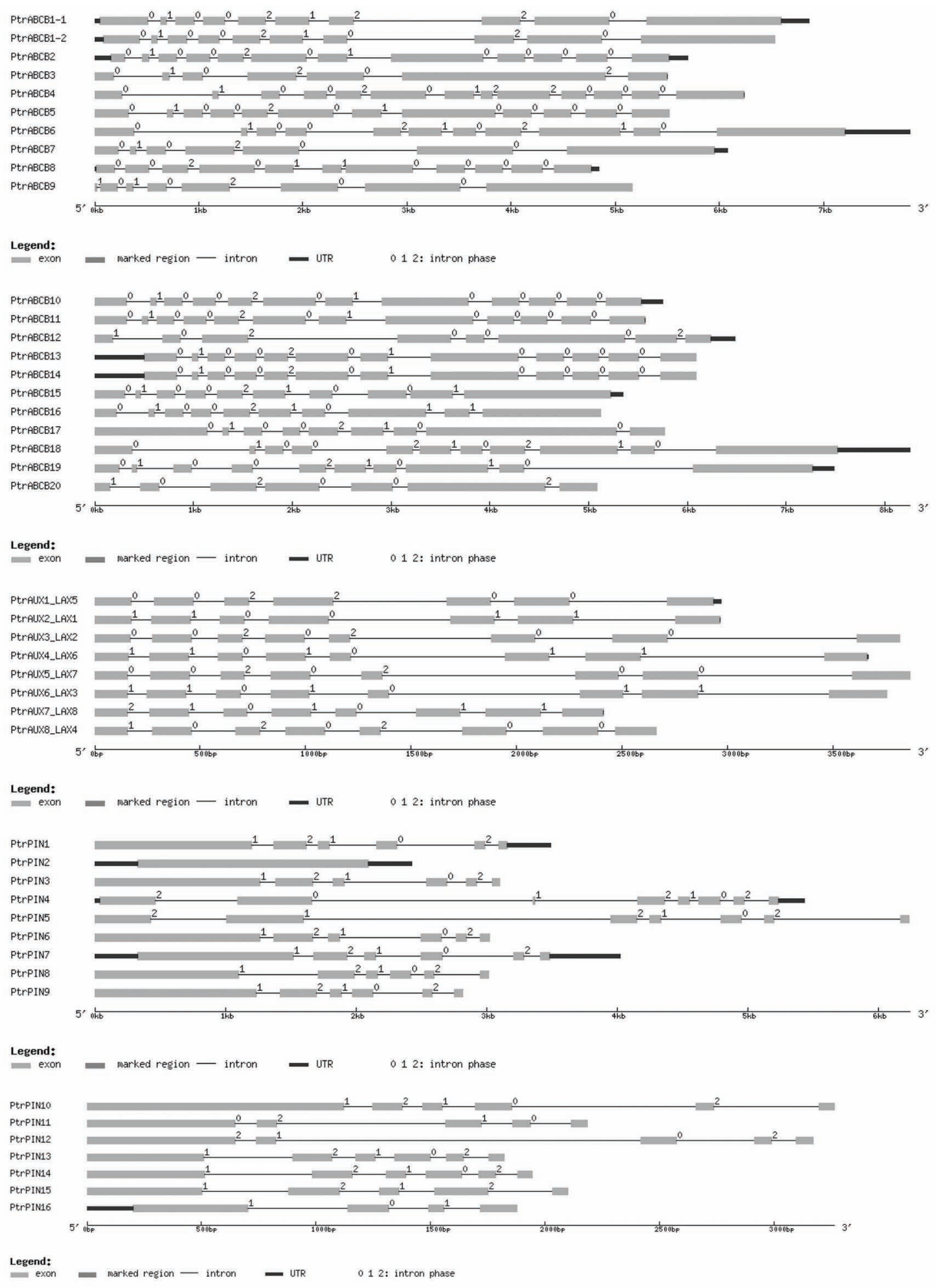

FIGURE A1 | Intron-exon structure of PIN, AUX/LAX, and ABCB genes from Populus trichocarpa. 


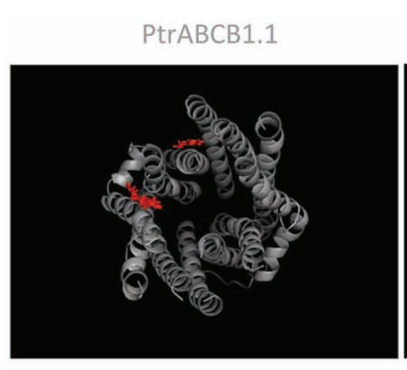

PtrABCB5

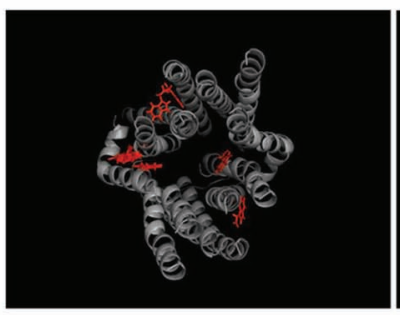

PtrABCB11

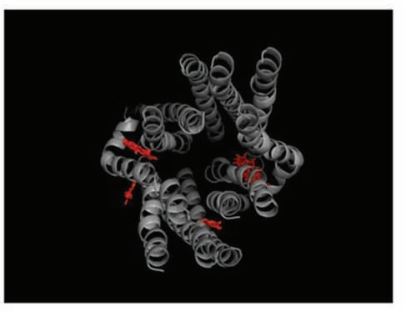

PtrABCB16

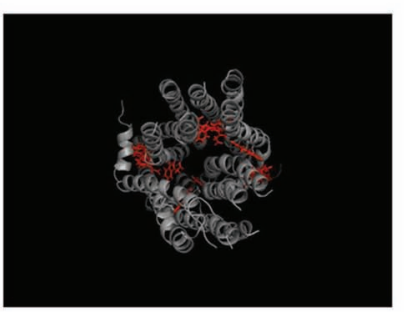

FIGURE A2 | Predicted model structures of putative auxin transport ABCBs from Populus trichocarpa. Tertiary protein structures have been generated using the python script Modeller 9v5. Predicted IAA docking sites are depicted in red.

\section{PtrABCB2}

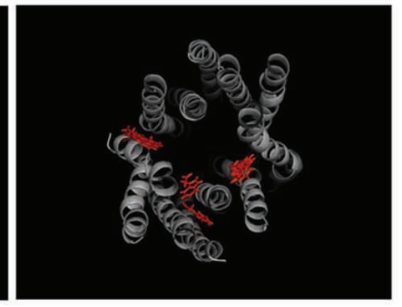

PtrABCB8

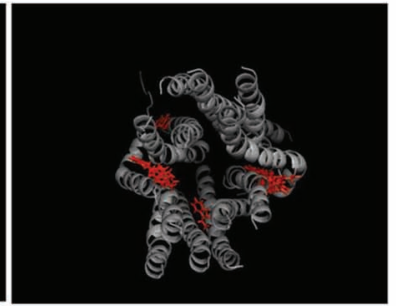

PtrABCB14

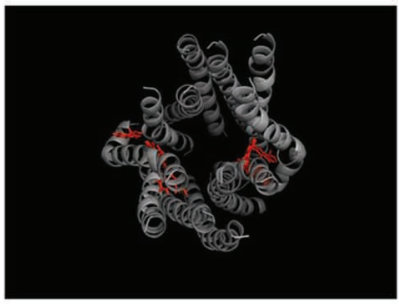

PtrABCB19

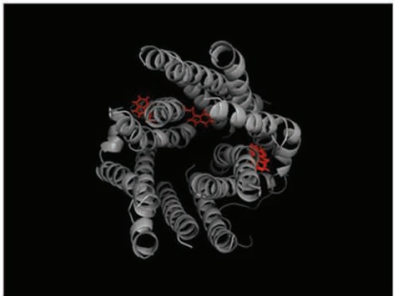


A

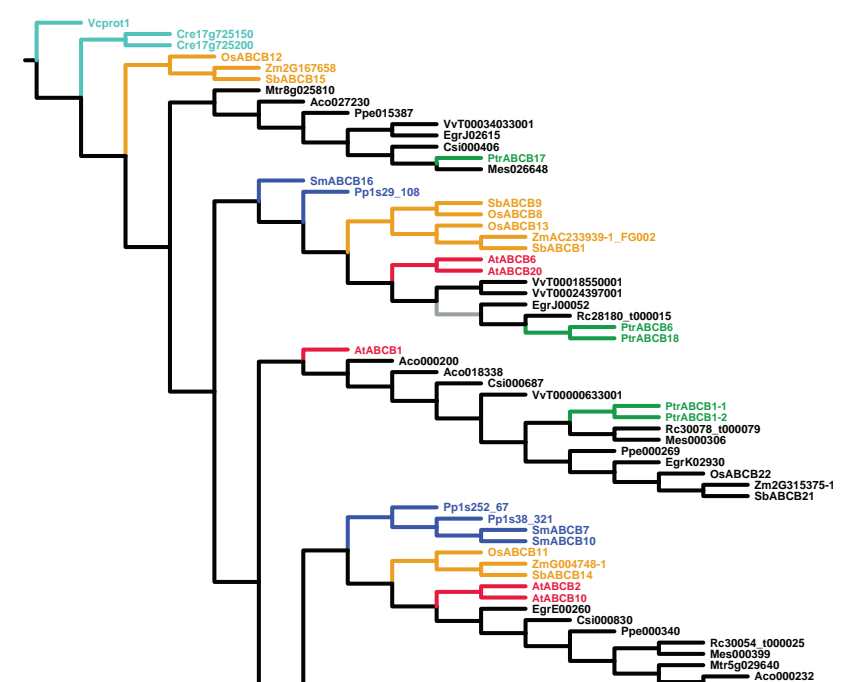

B

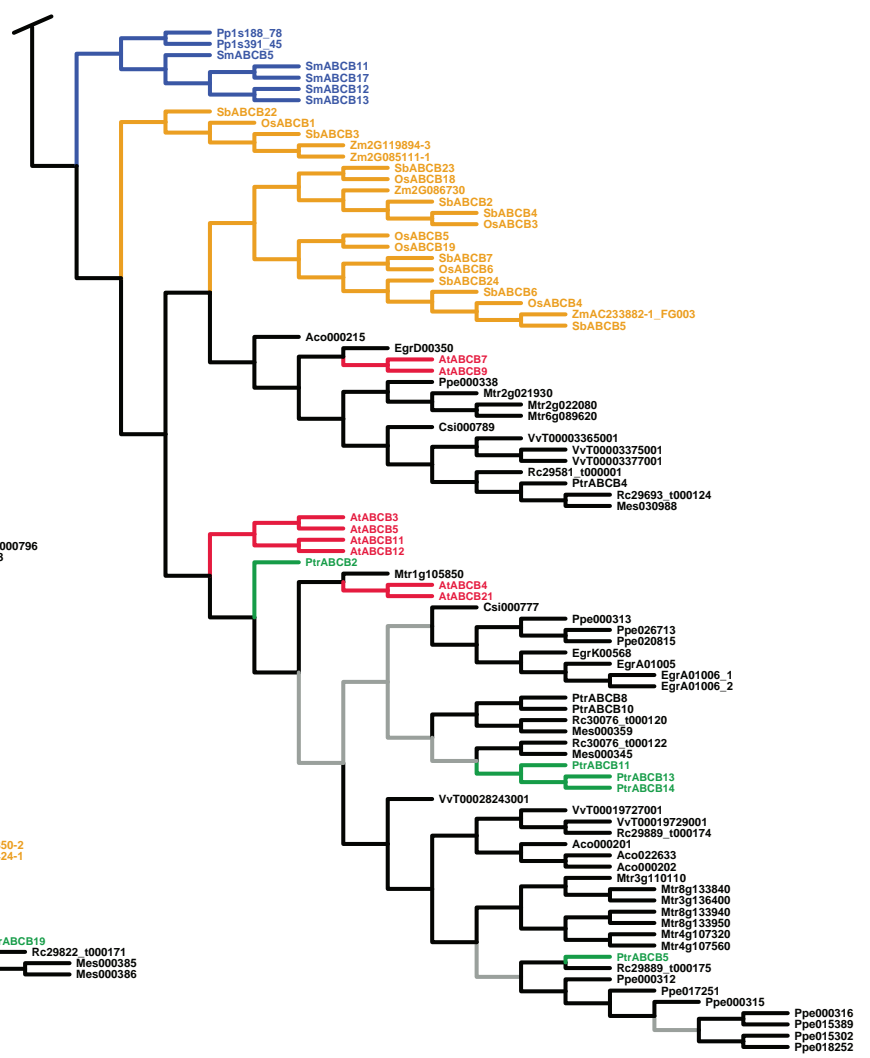

FIGURE A3 | Phylogeny of $A B C B$ genes from land plants, based upon coding sequences from the loci listed in Table A4, analyzed using dynamic homology under the parsimony criterion. Gray branches indicate nodes with bootstrap support lower than $50 \%$. Algal $A B C B$ s are colored light blue-green, basal land plants blue, Populus green, Arabidopsis red, and monocots yellow. Abbreviated names of each species are listed in Table A1. 
Table A1 | List of all species with their abbreviated names used in the present work.

\section{Species}

Abbreviation

Aquilegia caerulea

Aco

Arabidopsis thaliana

At

Chlamydomonas reinhardtii

Cre

Eucalyptus grandis

Egr

Manihot esculenta

Mes

Medicago truncatula

Mtr

Oryza sativa

Physcomitrella patens

Os

Populus tomentosa

$\mathrm{Pp}$

Populus tomentosa

Pto

Populus tremula $\times$ tremuloides

Ptt

Populus trichocarpa

Ptr

Prunus persica

Ppe

Ricinus communis

Rc

Selaginella moellendorffii

$\mathrm{Sm}$

Sorghum bicolor

$\mathrm{Sb}$

Vitis vinifera

Volvox carteri

Zea mays

Vv

$\mathrm{Vc}$

$\mathrm{Zm}$ 
Table A2 | List of putative auxin transport genes identified in the Populus trichocarpa genome.

\begin{tabular}{|c|c|c|}
\hline Genes & JGI v1.1 gene model & JGI v1.1 locus \\
\hline PtrPIN1 & estExt_fgenesh4_pg.C_LG_XV0366 & LG_XV:3955456-3958939 \\
\hline PtrPIN2 & estExt_Genewise1_v1.C_LG_XVI1213 & LG_XVI:2023747-2028247 \\
\hline PtrPIN4 & estExt_fgenesh4_pm.C_LG_V0399 & LG_V:12604974-12610191 \\
\hline PtrPIN5 & fgenesh4_pm.C_LG_II000334 & LG_II:4970467-4976705 \\
\hline PtrPIN8 & eugene3.00060333 & LG_VI:2296469-2299715 \\
\hline PtrPIN9 & fgenesh4_pm.C_LG_XVIII000434 & LG_XVIII:12913539-12916356 \\
\hline PtrPIN10 & fgenesh4_pm.C_LG_I000524 & LG_I:12290101-12293363 \\
\hline PtrPIN11 & estExt_fgenesh4_pg.C_870067 & scaffold_87:1004073-1006598 \\
\hline PtrPIN12 & fgenesh4_pg.C_LG_XIX000547 & LG_XIX:6900262-6903432 \\
\hline PtrAUX1/LAX5 & grail3.0023028402 & LG_VI:6769035-6772003 \\
\hline PtrAUX2/LAX1 & eugene3.00161081 & LG_XVI:10707443-10710997 \\
\hline PtrAUX3/LAX2 & estExt_fgenesh4_pg.C_LG_X1704 & LG_X:17003105-17007090 \\
\hline PtrAUX4/LAX6 & estExt_Genewise1_v1.C_LG_VIII1679 & LG_VIII:3795803-3800287 \\
\hline PtrAUX5/LAX7 & estExt_fgenesh4_pg.C_LG_IV1437 & LG_IV:15662320-15666183 \\
\hline PtrAUX6/LAX3 & grail3.0001031001 & LG_IX:2231536-2235747 \\
\hline PtrAUX7/LAX8 & estExt_fgenesh4_pg.C_LG_V0933 & LG_V:11098424-11101148 \\
\hline PtrAUX8/LAX4 & grail3.0003074001 & LG_II:6104679-6107343 \\
\hline PtrABCB1.1 & gw1.28.733.1 & scaffold_28:2297969-2304256 \\
\hline PtrABCB8 & estExt_fgenesh4_pm.C_LG_II0929 & LG_II:16965413-16970969 \\
\hline PtrABCB9 & fgenesh4_pg.C_LG_XVII000406 & LG_XVII:4919010-4924173 \\
\hline PtrABCB10 & eugene3.00140575 & LG_XIV:4755266-4761017 \\
\hline PtrABCB11 & eugene3.00140576 & LG_XIV:4765985-4771483 \\
\hline PtrABCB12 & gw1.XVIII.2596.1 & LG_XVIII:8860516-8866795 \\
\hline PtrABCB13 & eugene3.00140578 & LG_XIV:4778008-4781195 \\
\hline PtrABCB14 & estExt_fgenesh4_pm.C_LG_XIV0249 & LG_XIV:4781910-4787506 \\
\hline PtrABCB15 & fgenesh4_pm.C_LG_XV000001 & LG_XV:12903-18128 \\
\hline PtrABCB16 & fgenesh4_pm.C_LG_II000094 & LG_II:1130589-1135712 \\
\hline PtrABCB17 & eugene3.01580034 & scaffold_158:318976-324742 \\
\hline PtrABCB18 & fgenesh4_pg.C_LG_VIII000415 & LG_VIII:2748354-2755879 \\
\hline PtrABCB19 & estExt_fgenesh4_pg.C_LG_XVII0355 & LG_XVII:4160851-4168120 \\
\hline PtrABCB20 & fgenesh4_pm.C_LG_XI000351 & scaffold_11:16,395,988.0.16,402,087 \\
\hline
\end{tabular}


Table A2 | Continued

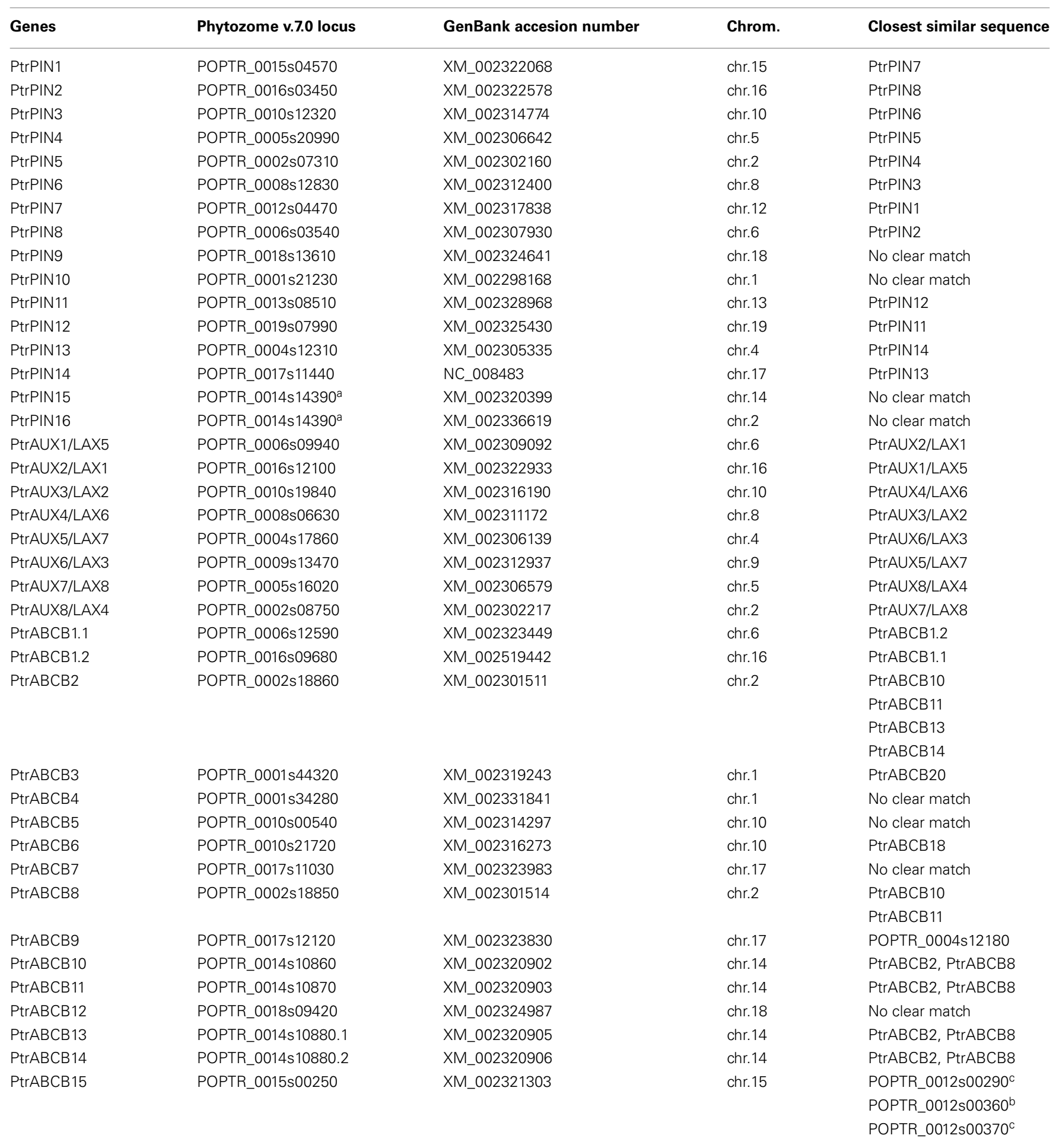




\section{Table A2 | Continued}

\begin{tabular}{lllll}
\hline Genes & Phytozome v.7.0 locus & GenBank accesion number & Chrom. & Closest similar sequence \\
\hline PtrABCB16 & POPTR_0002s02110 & XM_002301925 & chr.2 & No clear match \\
PtrABCB17 & POPTR_0001s16560 & XM_002331169 & chr.1 & No clear match \\
PtrABCB18 & POPTR_0008s05020 & XM_002311108 & chr.8 & PtrABCB6 \\
PtrABCB19 & POPTR_0017s11750 & XM_002323811 & chr.17 clear match \\
PtrABCB20 & POPTR_0011s13720 & XM_002316941 & chr.11 & PtrABCB3
\end{tabular}

Gene models, accession numbers, chromosome position, and the closest most similar match for each gene are reported.

aThese genes are distinct in GenBank but they retrieve the same entry in the phytozome database (www.phytozome.org).

${ }^{b}$ Very short protein classified as ATP-binding transporter.

' Uncharacterized conserved protein. 
Table A3 | Summary of the protein characteristics of the PIN, AUX/LAX, and ABCB families of Populus trichocarpa, Populus tomentosa, Populus tremula $\times$ tremuloides, and Arabidopsis.

\begin{tabular}{|c|c|c|c|c|}
\hline Gene & $\begin{array}{l}\text { length } \\
\text { cds (bp) }\end{array}$ & $\begin{array}{l}\text { Length } \\
\text { Protein (aa) }\end{array}$ & $\begin{array}{l}n \\
\text { TMHs }\end{array}$ & Type \\
\hline AtPIN1 & 1869 & 622 & 11 & Long \\
\hline AtPIN3 & 1923 & 640 & 10 & Long \\
\hline AtPIN4 & 1851 & 616 & 10 & Long \\
\hline AtPIN7 & 1860 & 619 & 10 & Long \\
\hline AtPIN8 & 1104 & 367 & 10 & Short \\
\hline PtrPIN1 & 1845 & 614 & 10 & Long \\
\hline PtrPIN2 & 1767 & 588 & 11 & Long \\
\hline PtrPIN3 & 1905 & 634 & 10 & Long \\
\hline PtrPIN8 & 1764 & 588 & 10 & Long \\
\hline PtrPIN9 & 1902 & 634 & 10 & Long \\
\hline PtrPIN10 & 1644 & 548 & 10 & Reduced \\
\hline PtrPIN11 & 1041 & 347 & 9 & Short \\
\hline PtrPIN12 & 1041 & 347 & 10 & Short \\
\hline PtrPIN13 & 1068 & 356 & 8 & Short \\
\hline PtrPIN14 & 1071 & 357 & 8 & Short \\
\hline PtrPIN15 & 1113 & 371 & 8 & Short \\
\hline PtrPIN16 & 912 & 304 & 6 & Short \\
\hline PttPIN1 & 1845 & 614 & 10 & Long \\
\hline PttPIN2 & 1767 & 588 & 10 & Long \\
\hline PtrAUX2/LAX1 & 1434 & 478 & 11 & \\
\hline PtrAUX3/LAX2 & 1422 & 474 & 11 & \\
\hline PtrAUX4/LAX6 & 1416 & 472 & 11 & \\
\hline PtrAUX5/LAX7 & 1476 & 492 & 11 & \\
\hline PtrAUX6/LAX3 & 1476 & 492 & 11 & \\
\hline PtrAUX7/LAX8 & 1395 & 465 & 11 & \\
\hline PtrAUX8/LAX4 & 1398 & 466 & 11 & \\
\hline PttLAX1 & 1434 & 477 & 10 & \\
\hline PttLAX2 & 1422 & 473 & 11 & \\
\hline PttLAX3 & 1476 & 491 & 11 & \\
\hline PtoAUX1 & 1434 & 477 & 10 & \\
\hline AtABCB1 & 3861 & 1286 & 12 & \\
\hline AtABCB2 & 3822 & 1273 & 12 & \\
\hline AtABCB3 & 3690 & 1229 & 11 & \\
\hline AtABCB4 & 3861 & 1286 & 9 & \\
\hline AtABCB5 & 3693 & 1230 & 9 & \\
\hline AtABCB6 & 4224 & 1407 & 13 & \\
\hline
\end{tabular}


Table A3 | Continued

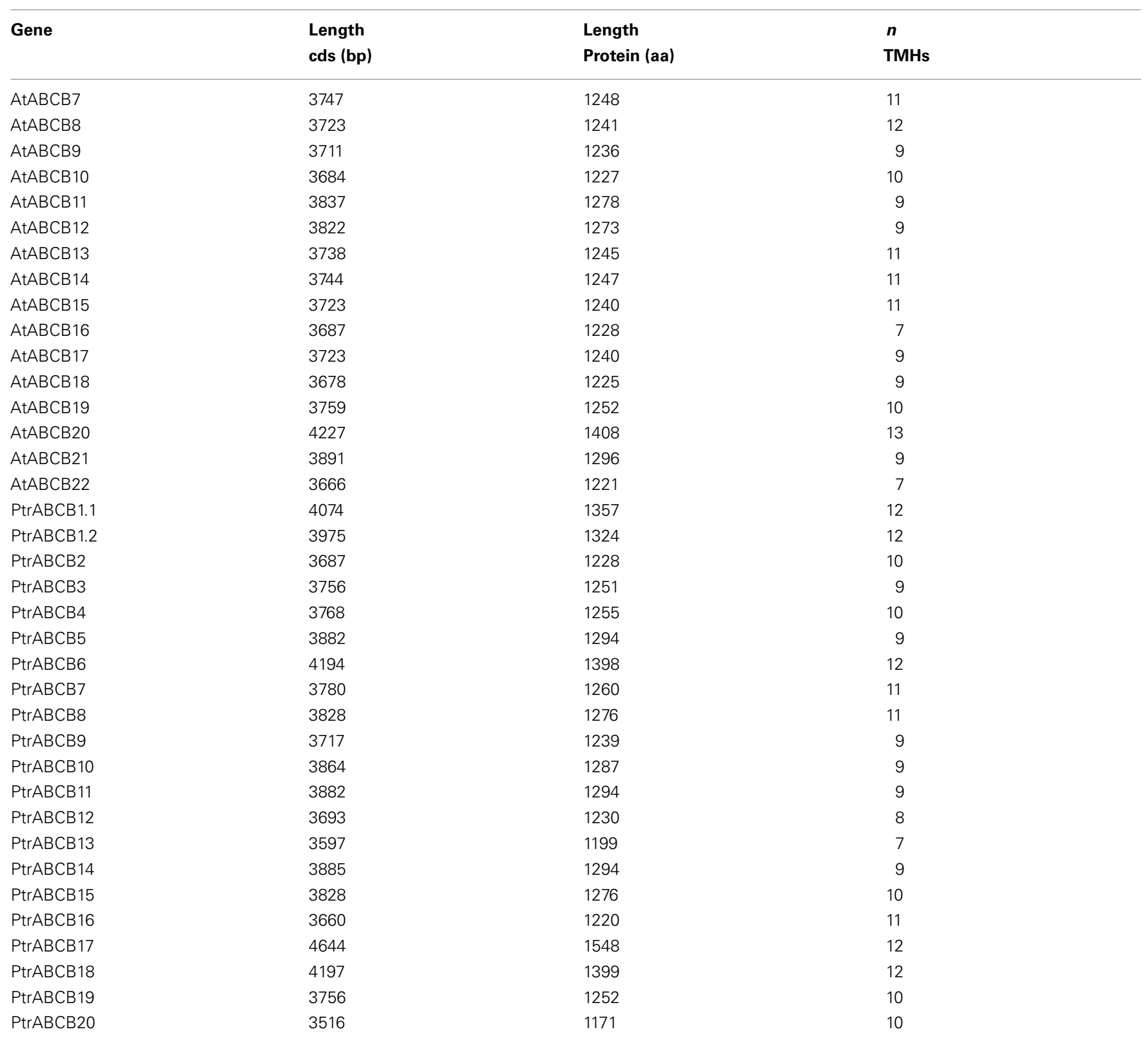

All proteins are classified according to their sequence length, number of predicted transmembrane helices, and length of the central hydrophilic loop (short, reduced, long). 
Table A4 | List of all the sequences used in the reconstruction of PIN, $A U X / L A X$, and $A B C B$ families phylogenies.

Phytozome database locus or GenBank accession number

Assigned name

\begin{tabular}{|c|c|}
\hline \multicolumn{2}{|l|}{ ABCBs } \\
\hline ppa000359m.g & Ppe000359 \\
\hline ppa000340m.g & Ppe000340 \\
\hline ppa000313m.g & Ppe000313 \\
\hline ppa000316m.g & Ppe000316 \\
\hline ppa023953m.g & Ppe023953 \\
\hline ppa000363m.g & Ppe000363 \\
\hline ppa015387m.g & Ppe015387 \\
\hline ppa015389m.g & Ppe015389 \\
\hline ppa017251m.g & Ppe017251 \\
\hline ppa023915m.g & Ppe023915 \\
\hline ppa000338m.g & Ppe000338 \\
\hline ppa0208157m.g & Ppe020815 \\
\hline POPTR_0006s12590 & PtrABCB11 \\
\hline POPTR_0016s09680 & PtrABCB12 \\
\hline POPTR_0002s18860 & PtrABCB2 \\
\hline POPTR_0001s44320 & PtrABCB3 \\
\hline POPTR_0001s34280 & PtrABCB4 \\
\hline POPTR_0010s00540 & PtrABCB5 \\
\hline POPTR_0010s21720 & PtrABCB6 \\
\hline POPTR_0017s11030 & PtrABCB7 \\
\hline POPTR_0002s18850 & PtrABCB8 \\
\hline POPTR_0002s02110 & PtrABCB16 \\
\hline POPTR_0001s16560 & PtrABCB17 \\
\hline POPTR_0008s05020 & PtrABCB18 \\
\hline POPTR_0017s11750 & PtrABCB19 \\
\hline POPTR_0011s13720 & PtrABCB20 \\
\hline GRMZM2G315375_T01 & Zm2G315375-1 \\
\hline GRMZM2G085236_T01 & Zm2G085236-1 \\
\hline GRMZM2G085236_T02 & ZmG085236-2 \\
\hline GRMZM2G004748_T01 & ZmG004748-1 \\
\hline GRMZM2G119894_T01 & Zm2G119894-1 \\
\hline GRMZM2G119894_T03 & Zm2G119894-3 \\
\hline GRMZM2G086730_T01 & Zm2G086730 \\
\hline AC233882.1_FGT003 & ZmAC233882-1_FG003 \\
\hline GRMZM2G025860_T01 & Zm2G025860 \\
\hline GRMZM2G167658_T01 & Zm2G167658 \\
\hline GRMZM2G111462_T01 & Zm2G111462 \\
\hline GRMZM2G085111_T02 & Zm2G085111-1 \\
\hline
\end{tabular}


Table A4 | Continued

Phytozome database locus or GenBank accession number

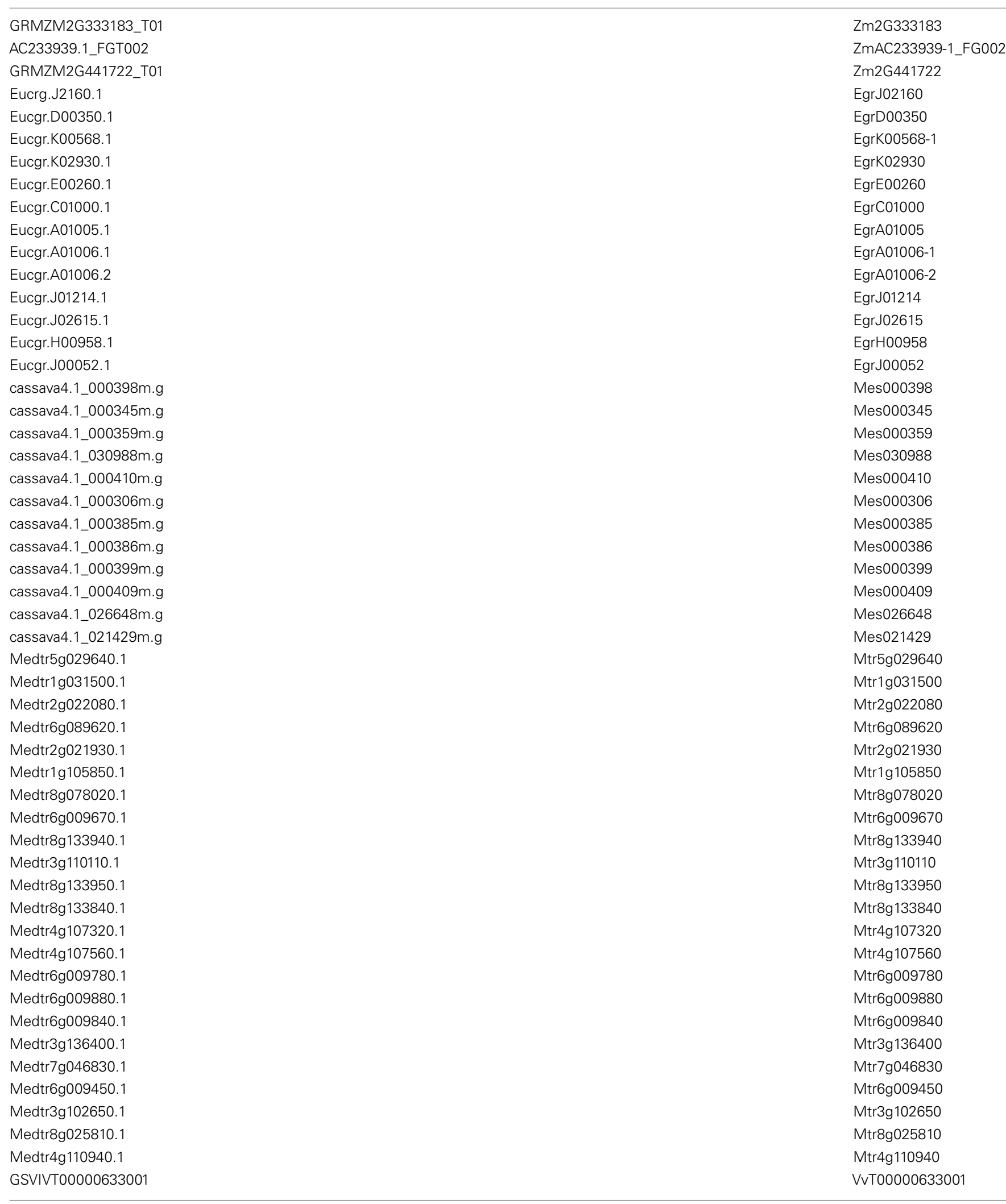

\section{Assigned name}

Zm2G333183

EgrD00350

EgrC01000

EgrA01005

EgrA01006-

EgrA01006-2

EgrJ01214

Mes000359

Mes030988

Mes000410

Mtr5g029640

Mtr1g031500

Mtr1g105850

Mtr8g078020

Mtr6g009670

Mtr8g133940

Mtr3g110110

Mtr4g10756

Mtr6g009840

Mtr3g136400 
Table A4 | Continued

Phytozome database locus or GenBank accession number

Assigned name

GSVIVT00003365001

GSVIVT00003375001

GSVIVT00003377001

GSVIVT00014386001

GSVIVT00016667001

GSVIVT00018550001

GSVIVT00019727001

GSVIVT00019729001

GSVIVT00020929001

GSVIVT00024397001

GSVIVT00028243001

GSVIVT00030719001

GSVIVT00034033001

GSVIVT00037129001

Sb01g039110.1

Sb02g019540.1

Sb03g011860.1

Sb03g023740.1

Sb03g031990.1

Sb03g032000.1

Sb03g032030.1

Sb03g033290.1

Sb03g047490.1

Sb04g006087.1

Sb04g006090.1

Sb04g006100.1

Sb04g022480.1

Sb04g031170.1

Sb06g001440.1

Sb06g018860.1

Sb06g020350.1

Sb06g030350.1

Sb07g003510.1

Sb07g003520.1

Sb07g023730.1

Sb09g002940.1

Sb09g027320.1

Sb09g027330.1

e_gw1.13.597.1

fgenesh1_pm.C_scaffold_6000062

fgenesh2_pg.C_scaffold_13000013

e_gw1.6.146.1

estExt_Genewise1Plus.C_350372

fgenesh1_pm.C_scaffold_42000045

e_gw1.0.369.1

fgenesh2_pg.C_scaffold_9000128

estExt_Genewise1.C_210058

fgenesh1_pm.C_scaffold_2000054

e_gw1.73.37.1

estExt_Genewise1Plus.C_90010

e_gw1.0.1863.1

e_gw1.22.307.1

VvT00003365001

VvT00003375001

VvT00003377001

VvT00014386001

VvT00016667001

VvT00018550001

VvT00019727001

VvT00019729001

VvT00020929001

VvT00024397001

VvT00028243001

VvT00030719001

VvT00034033001

VvT00037129001

SbABCB1

SbABCB2

SbABCB3

SbABCB4

SbABCB5

SbABCB6

SbABCB7

SbABCB8

SbABCB9

SbABCB10

SbABCB11

SbABCB12

SbABCB13

SbABCB14

SbABCB15

SbABCB16

SbABCB17

SbABCB18

SbABCB19

SbABCB20

SbABCB21

SbABCB22

SbABCB23

SbABCB24

SmABCB1

SmABCB2

SmABCB3

SmABCB4

SmABCB5

SmABCB6

SmABCB7

SmABCB8

SmABCB9

SmABCB10

SmABCB11

SmABCB12

SmABCB13

SmABCB14

(Continued) 
Table A4 | Continued

Phytozome database locus or GenBank accession number

Assigned name

fgenesh1_pm.C_scaffold_0000169

estExt_Genewise1.C_00569

e_gw1.73.196.1

fgenesh1_pm.C_scaffold_15000068

LOC_Os01g18670.1

LOC_Os01g35030.1

LOC_Os01g50080.1

LOC_Os01g50100.1

LOC_Os01g50160.1

LOC_Os01g52550.1

LOC_Os01g74470.1

LOC_Os02g09720.1

LOC_Os02g46680.1

LOC_Os03g08380.1

LOC_Os03g17180.1

LOC_Os04g40570.1

LOC_Os05g47490.1

LOC_Os05g47500.1

LOC_Os08g05690.1

LOC_Os08g05710.1

LOC_Os08g45030.1

Rco30078.t000079

Rco30054.t000025

Rco30076.t000120

Rco30076.t000122

Rco28180.t000015

Rco30170.t000796

Rco29581.t000001

Rco29693.t000124

Rco29822.t000171

Rco29889.t000174

Rco29889.t000175

Pp1s252_67V6.1

Pp1s38_321V6.1

Pp1s28_282V6.1

Pp1s173_145V6.1

Pp1s1_780V2.1

Pp1s397_2V6.1

Pp1s188_78V6.1

Pp1s391_45V6.1

Pp1s338_12V6.1

Pp1s29_108V2.1

Vc_estExt_fgenesh4_pg.C_30286

Cre17.g725200

Cre17.g725150

AT2G36910

AT4G25960

AT4G01820

AT2G47000

AT4G01830

AT2G39480

AT5G46540

SmABCB15

SmABCB16

SmABCB17

SmABCB18

OsABCB1

OsABCB3

OsABCB4

OsABCB5

OsABCB6

OsABCB7

OsABCB8

OsABCB9

OsABCB11

OsABCB12

OsABCB13

OsABCB15

OsABCB18

OsABCB19

OsABCB20

OsABCB21

OsABCB22

Rc30078_t000079

Rc30054_t000025

Rc30076_t000120

Rc30076_t000122

Rc28180_t000015

Rc30170_t000796

Rc29581_t000001

Rc29693_t000124

Rc29822_t000171

Rc29889_t000174

Rc29889_t000175

Pp1s252_67

Pp1s38_321

Pp1s28_282

Pp1s173_145

Pp1s1_780

Pp1s397_2

Pp1s188_78

Pp1s391_45

Pp1s338_12

Pp1s29_108

VcProt1

Cre17_g725200

Cre17_g725150

AtABCB1

AtABCB2

AtABCB3

AtABCB4

AtABCB5

AtABCB6

AtABCB7 
Table A4 | Continued

Phytozome database locus or GenBank accession number

Assigned name

AT3G30875

AtABCB8

AT4G18050

AtABCB9

AT1G10680

AtABCB10

At1g02520

AtABCB11

AT1G02530

AtABCB12

AT1G27940

AtABCB13

AT1G28010

AtABCB14

AT3G28345

AtABCB15

АT3G28360

AtABCB16

АT3G28380

AtABCB17

AT3G28390

AtABCB18

AT3G28860

AtABCB19

AT3G55320

AtABCB20

AT3G62150

AtABCB21

AT3G28415

AtABCB22

orange $1.1 \mathrm{~g} 000851 \mathrm{~m} . \mathrm{g}$

Csi_g000851

orange $1.1 \mathrm{~g} 000777 \mathrm{~m} . \mathrm{g}$

Csi_g000777

orange $1.1 \mathrm{~g} 000789 \mathrm{~m} . \mathrm{g}$

Csi_g000789

orange $1.1 \mathrm{~g} 000909 \mathrm{~m} . \mathrm{g}$

Csi_g000909

orange $1.1 \mathrm{~g} 000830 \mathrm{~m} . \mathrm{g}$

Csi_g000830

orange $1.1 \mathrm{~g} 000406 \mathrm{~m} . \mathrm{g}$

Csi_g000406

orange $1.1 \mathrm{~g} 000687 \mathrm{~m} . \mathrm{g}$

Csi_g000687

orange $1.1 \mathrm{~g} 000856 \mathrm{~m} . \mathrm{g}$

Csi_g000856

AcoGoldSmith_v1.000232m.g

Aco000232

AcoGoldSmith_v1.022827m.g

Aco022827

AcoGoldSmith_v1.027230m.g

Aco027230

AcoGoldSmith_v1.000200m.g

Aco000200

AcoGoldSmith_v1.018338m.g

Aco018338

AcoGoldSmith_v1.000314m.g

Aco000314

AcoGoldSmith_v1.022346m.g

Aco022346

AcoGoldSmith_v1.026987m.g

Aco026987

AcoGoldSmith_v1.022633m.g

Aco022633

AcoGoldSmith_v1.000202m.g

Aco000202

AcoGoldSmith_v1.000201m.g

Aco000201

AcoGoldSmith_v1.000230m.g

Aco000230

AcoGoldSmith_v1.000215m.g

Aco000215

AcoGoldSmith_v1.000236m.g

Aco000236

AcoGoldSmith_v1.000229m.g

Aco000229

\section{AUX/LAXs}

ppa005323m.g

ppa005057m.g

ppa004949m.g

ppa004865m.g

POPTR_0006s09940

POPTR_0016s12100

POPTR_0010s19840

Ppe005323

Ppe005057

Ppe004949

Ppe004865

PtrAUX1/LAX5

PtrAUX2/LAX1

PtrAUX3/LAX2

POPTR_0008s06630

PtrAUX4/LAX6

POPTR_0004s17860

PtrAUX5/LAX7

POPTR_0009s13470

PtrAUX6/LAX3

POPTR_0005s 16020

PtrAUX7/LAX8

POPTR_0002s08750

PtrAUX8/LAX4

GRMZM2G067022_T01

Zm2G067022 
Table A4 | Continued

Phytozome database locus or GenBank accession number

Assigned name

GRMZM2G127949_T01

GRMZM2G045057_T01

GRMZM2G149481_T01

GRMZM2G129413_T01

Eucgr.F03758.1

Eucgr.K02992.2

Eucgr.G03044.2

Eucgr.G01769.2

Eucgr.A00514.2

cassava4.1_006838m.g

cassava4.1_006423m.g

cassava4.1_006788m.g

cassava4.1_006570m.g

cassava4.1_006783m.g

cassava4.1_006474m.g

cassava4.1_007093m.g

Medtr3g024670.1

Medtr3g097960.1

Medtr5g089600.1

GSVIVT01008917001

GSVIVT01024054001

GSVIVT01032855001

GSVIVT01033986001

Sb01g026240.1

Sb01g041270.1

Sb03g040320.1

Sb05g004250.1

Sb09g021990.1

estExt_Genewise1Plus.C_20968

estExt_fgenesh2_pg.C_50586

LOC_Os01g63770.1

LOC_Os03g14080.1

LOC_Os05g37470.1

LOC_Os10g05690.1

LOC_Os11g06820.1

Rco29669.t000030

Rco29741.t000002

Rco29908.t000197

Rco29969.t000004

Pp1s90_46V6.1

Pp1s213_89V6.1

Pp1s211_67V6.1

AT2G38120.1

AT5G01240.1

AT2G21050.1

AT1G77690.1

orange1.1g011392m.g

orange $1.1 \mathrm{~g} 011022 \mathrm{~m} . \mathrm{g}$

orange $1.1 \mathrm{~g} 012371 \mathrm{~m} . \mathrm{g}$

orange1.1g011966m.g

AcoGoldSmith_v1.004219m.g

AcoGoldSmith_v1.004342m.g

Zm2G127949

Zm2G045057

Zm2G149481

Zm2G129413

EgrF03758_1

EgrK02992_2

EgrG03044_2

EgrG01769_2

EgrA00514_2

Mes006838

Mes006423

Mes006788

Mes006570

Mes006783

Mes006474

Mes007093

Mtr3g024670

Mtr3g097960

Mtr5g089600

VvT01008917001

VvT01024054001

VvT01032855001

VvT01033986001

SbLAX1

SbLAX2

SbLAX3

SbLAX4

SbLAX5

SmAUX1

SmAUX2

OsLAX1

OsLAX2

OsLAX3

OsLAX4

OsLAX5

Rc29669_t000030

Rc29741_t000002

Rc29908_t000197

Rc29969_t000004

Pp1s90_46

Pp1s213_89

Pp1s211_67

AtAUX1

AtLAX1

AtLAX2

AtLAX3

Csi_g011392

Csi_g011022

Csi_g012371

Csi_g011966

Aco004219

Aco004342

(Continued)

Frontiers in Plant Science | Plant Physiology 
Table A4 | Continued

Phytozome database locus or GenBank accession number

Assigned name

AcoGoldSmith_v1.003895m.g

Aco003895

AY864733

Pto-AY864733

AF115543

Ptt-AF115543

\section{PINs}

ppa022797m.g

ppa003159m.g

Ppe022797

ppa024134m.g

ppa002528m.g

ppa025174m.g

ppa002944m.g

ppa021573m.g

ppa007621m.g

POPTR_0015s04570

Ppe003159

Ppe024134

Ppe002528

Ppe025174

Ppe002944

Ppe021573

Ppe007621

POPTR_0016s03450

PtrPIN1

POPTR_0010s12320

PtrPIN2

POPTR_0005s20990

PtrPIN3

POPTR_0002s07310

PtrPIN4

POPTR_0008s12830

PtrPIN5

POPTR_0012s04470

PtrPIN6

POPTR_0006s03540

PtrPIN7

PtrPIN8

POPTR_0018s13610

PtrPIN9

POPTR_0001s21230

PtrPIN10

POPTR_0013s08510

PtrPIN11

POPTR_0019s07990

PtrPIN12

POPTR_0004s12310

PtrPIN13

POPTR_0017s11440

PtrPIN14

POPTR_0014s14390

PtrPIN15

XM_002336619.1

PtrPIN16

ZmPIN1a_GRMZM2G098643

ZmPIN1a

ZmPIN1b_GRMZM2G074267

ZmPIN1b

ZmPIN1c_GRMZM2G149184

ZmPIN1c

ZmPIN1d_GRMZM2G171702_T01

ZmPIN1d

ZmPIN2

ZmPIN5a-GRMZM2G025742

ZmPIN2

ZmPIN5b-GRMZM2G148648

ZmPIN5a

ZmPIN5b

ZmPIN5c-GRMZM2G040911

ZmPIN5c

ZmPIN8_GRMZM5G839411

ZmPIN8

ZmPIN9_GRMZM5G859099

ZmPIN9

ZmPIN10a-GRMZM2G126260

ZmPIN10a

ZmPIN10b-GRMZM2G160496

ZmPIN10b

Eucgr.F04265.1

EgrF04265_1

Eucgr.K02271.1

Eucgr.G02187.1

Eucgr.G02549.1

Eucgr.B01406.1

Eucgr.B02902.1

Eucgr.B00948.1

EgrK02271_1

EgrG02187_1

EgrG02549_1

EgrB01406_1

EgrB02902_1

EgrB00948_1

Eucgr.C00078.1

EgrC00078_1

Eucgr.A02229.1

EgrA02229_1

Eucgr.H01390.1

EgrH01390_1

Eucgr.H01391.1

EgrH01391_1

Eucgr.I01919.1

Egrl01919_1 
Table A4 | Continued

Phytozome database locus or GenBank accession number

Assigned name

\section{Eucgr.G02548.1}

Eucgr.B01405.1

Eucgr.B01403.1

Eucgr.H01382.1

cassava4.1_003807m.g

cassava4.1_030090m.g

cassava4.1_029078m.g

cassava4.1_003367m.g

cassava4.1_006998m.g

cassava4.1_026579m.g

cassava4.1_003794m.g

cassava4.1_029063m.g

cassava4.1_033391m.g

cassava4.1_010688m.g

cassava4.1_010607m.g

Medtr2g043210

Medtr4g154810

Medtr6g083450

Medtr7g008720

Medtr7g089430

Medtr7g106430

Medtr8g130020

Medtr8g130040

MtrAAM55297

MtrAY115838

MtrAAT48627

GSVIVT00014302001

GSVIVT00017824001

GSVIVT00020886001

GSVIVT00023254001

GSVIVT00023255001

GSVIVT00025093001

GSVIVT00025108001

GSVIVT00030482001

GSVIVT00031315001

Sb02g029210.1

Sb03g029320.1

Sb03g032850.1

Sb03g037350.1

Sb03g043960.1

Sb04g028170.1

Sb05g002150.1

Sb07g026370.1

Sb10g004430.1

Sb10g008290.1

Sb10g026300.1

e_gw1.26.13.1

e_gw1.59.169.1

fgenesh1_pm.C_scaffold_9000007

fgenesh1_pm.C_scaffold_59000022

estExt_fgenesh1_pm.C_500006

EgrG02548_1

EgrB01405_1

EgrB01403_1

EgrH01382_1

Mes003807

Mes030090

Mes029078

Mes003367

Mes006998

Mes026579

Mes003794

Mes029063

Mes033391

Mes010688

Mes010607

Mtr2g043210

Mtr4g154810

Mtr6g083450

Mtr7g008720

Mtr7g089430

Mtr7g106430

Mtr8g130020

Mtr8g130040

MtrAAM55297

MtrAY115838

MtrAAT48627

VvT00014302001

VvT00017824001

VvT00020886001

VvT00023254001

VvT00023255001

VvT00025093001

VvT00025108001

VvT00030482001

VvT00031315001

SbPIN1

SbPIN2

SbPIN3

SbPIN4

SbPIN5

SbPIN6

SbPIN7

SbPIN8

SbPIN9

SbPIN10

SbPIN11

Sm102666

Sm119024

Sm231064

Sm234325

Sm268490

(Continued) 
Table A4 | Continued

Phytozome database locus or GenBank accession number

Assigned name

e_gw1.21.81.1

Os01g45550.1

Sm99301

Os01g51780

OsPIN10a

Os01g58860

OsPIN8

Os01g69070

OsPIN9

Os02g50960.1

OsPIN5a

Os05g50140

OsPIN1b

Os06g12610

OsPIN10b

Os06g44970

OsPIN1a

Os08g41720

OsPIN2

Os09g32770

OsPIN5b

Os11g04190

OsPIN5c

Os12g04000

OsPIN1c

Rco27985.t000045

OsPIN1d

Rco29662.t000026

Rc27985_t000045

Rco29816.t000014

Rco30180.t000054

Rco29822.t000149

Rco30128.t000486

Pp1s10_17V6.1

Pp1s18_186V6.1

Rc29662_t000026

Rc29816_t000014

Rc30180_t000054

Rc29822_t000149

Rc30128_t000486

PpPIN1A

PpPIN1B

Pp1s32_43V6.1

PpPIN1C

Pp1s79_126V6

AT1G73590

PpPIN1D

AT5G57090

AtPIN1

AT1G70940

AtPIN2

AtPIN3

AT2G01420

AtPIN4

AT5G16530

AtPIN5

AT1G77110

AtPIN6

AT1G23080

AtPIN7

AT5G15100

AtPIN8

orange $1.1 \mathrm{~g} 006199 \mathrm{~m} . \mathrm{g}$

Csi_g006199

orange $1.1 \mathrm{~g} 007826 \mathrm{~m} . \mathrm{g}$

Csi_g007826

Csi_g036474

Csi_g041301

orange $1.1 \mathrm{~g} 041301 \mathrm{~m} . \mathrm{g}$

Csi_g048649

Csi_g035534

Csi_g007420

Csi_g018360

Csi_g019021

Aco001931

AcoGoldSmith_v1.001931m.g

Aco018694

Aco018139

Aco016169

Aco007499

Aco021242

PtoPIN1-like

PttPIN1

PttPIN2

AF190881

PttPIN3

AF515435 
Table A5 | List of all primers used in the present work.

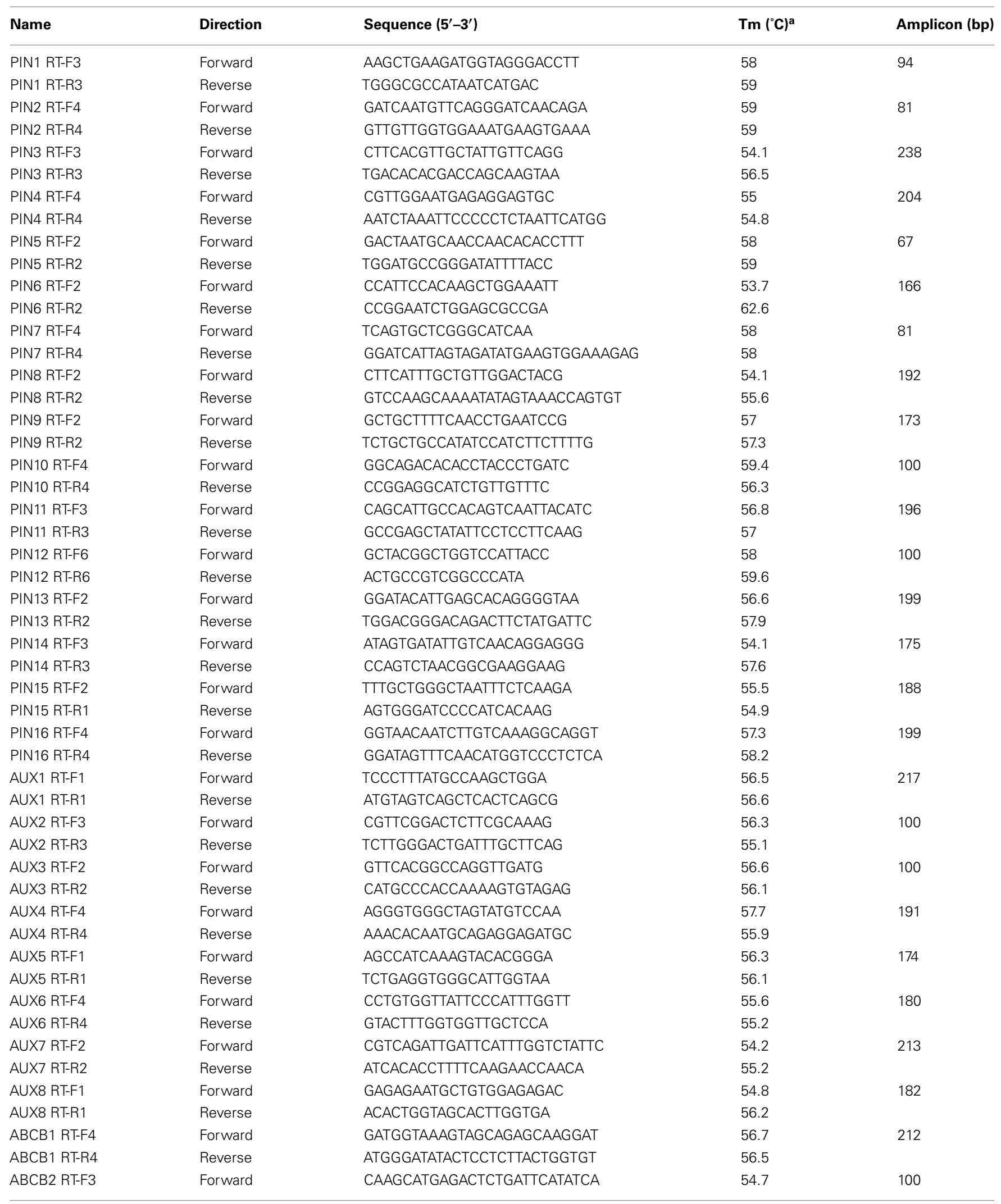


Table A5 | Continued

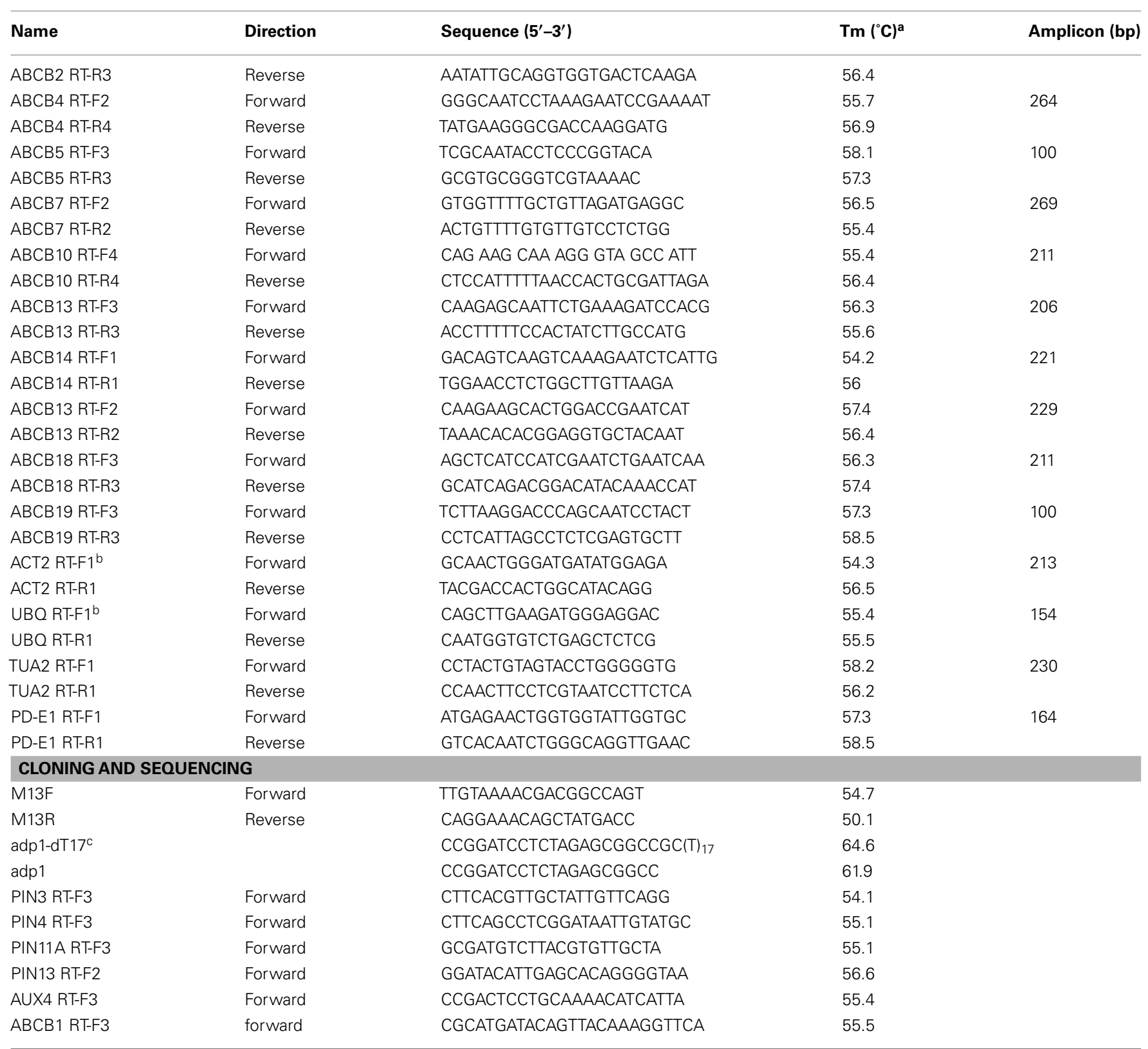

a Melting temperatures were calculated with the online tool OlygoAnalyzer v.3.1 from Integrated DNA Technologies.

${ }^{b}$ These primer pairs have been first published in Secchi et al. (2009).

'This primer sequence has been first published in Kramer et al. (1998). 